\title{
SCATTERING OF SOLUTIONS AND STABILITY OF SOLITARY WAVES FOR THE GENERALIZED BBM-ZK EQUATION*
}

\author{
AMIN ESFAHANI ${ }^{\dagger}$
}

\begin{abstract}
In this paper, a two-dimensional version of the BBM equation will be considered. The existence and scattering of global small amplitude solutions to this equation will be studied. The orbital stability of solitary wave solutions of this equation will be also investigated.
\end{abstract}

Key words. BBM-ZK equation, scattering, orbital stability.

AMS subject classifications. 35Q35, 35Q20, 76B15, 76B25, 35B35.

\section{Introduction}

In [2], Benjamin, Bona, and Mahony derived the following alternative to the KdV equation model for the propagation of long, weakly nonlinear one-dimensional waves:

$$
u_{t}+u_{x}-u_{x x t}+u u_{x}=0,
$$

where $u$ is a real-valued function. The idea behind the derivation of (1.1) (so-called $\mathrm{BBM}$ equation) is that the dispersion of the linearized equation at zero, while being equivalent to that of the linearized $\mathrm{KdV}$ equation for long waves, does not possess the bad short wave behavior of the $\mathrm{KdV}$ dispersion $\omega(k)=k-k^{3}$ (see [2] for a very lucid discussion of the shortcomings of the $\mathrm{KdV}$ equation with respect to modeling).

A natural weakly two dimensional generalization of (1.1) is so-called BBM-ZK equation $[1,13,22,34]$

$$
u_{t}+\varepsilon u_{x}-\left(u_{x t}+\beta u_{y y}\right)_{x}+u u_{x}=0,
$$

where $\beta \in \mathbb{R}^{*}, \varepsilon>0$, and $u=u(x, y, t)$ is a real-valued function. One can easily observe that the dispersion relation of (1.2) is a good approximation of that of the ZakharovKuznetsov (ZK) equation

$$
u_{t}+\left(u_{x x}+u_{y y}\right)_{x}+u u_{x}=0
$$

which can be considered as a two dimensional version of the KdV equation

$$
u_{t}+u_{x x x}+u u_{x}=0 .
$$

The motivation of this work is to compare the behaviors of the solutions of the twodimensional versions of (1.1) and (1.4). In [16], Faminskii considered the initial value problem associated with the ZK equation. He showed the local and global wellposedness for initial data in $H^{m}\left(\mathbb{R}^{2}\right)$, with $m \in \mathbb{N}$. In [5], Biagioni and Linares dealt with the case $p=2$ for the generalized Zakharov-Kuznetsov (gZK) equation,

$$
u_{t}+\left(u_{x x}+u_{y y}\right)_{x}+u^{p} u_{x}=0
$$

and proved the local well-posedness for data in $H^{1}\left(\mathbb{R}^{2}\right)$. By considering the cases $p=1$ and $p=2$, Linares and Pastor [25] improved the results in [5, 16] by showing

${ }^{*}$ Received: March 12, 2012; accepted (in revised form): March 8, 2013. Communicated by Paul Milewski.

${ }^{\dagger}$ School of Mathematics and Computer, Science, Damghan University, Damghan, P.O. Box 36715364, Iran (amin@impa.br, esfahani@du.ac.ir). 
that the initial value problem associated with equation (1.3) is locally well-posed for initial data in $H^{s}\left(\mathbb{R}^{2}\right), s>3 / 4$. Moreover they also proved the global well-posedness in $H^{1}\left(\mathbb{R}^{2}\right)$, for $p=2$, via a standard method by using the unique ground state of (1.5). Recently, the local and global well-posedness and a nonlinear scattering result in the energy space, for the case $p \geq 3$, have been investigated in $[17,26]$. More precisely, the authors in [17] obtained the local well-posedness of $(1.5)$ in $H^{s}\left(\mathbb{R}^{2}\right)$ for $s>3 / 4$ if $2 \leq p \leq 8$, and $s>1-2 / p$ if $p>8$. They also showed that solutions of (1.5) with small initial data decay to zero like $(1+|t|)^{-\frac{2 p}{3(p+1)}}$ in $L^{2(p+1)}\left(\mathbb{R}^{2}\right)$. Recently Ribaud and Vento in [30] improved these results to $s>1 / 4$ if $p=2, s>5 / 12$ if $p=3$ and $s>1-2 / p$ if $p \geq 4$; see also results of [29] for the three dimensional case of (1.5). It is noteworthy that the Cauchy problem for the ZK equation in the Bourgain-type spaces $[9,10,11]$ seems not to work. It should also be noted that questions of existence and orbital stability of solitary wave solutions of (1.5) were addressed by de Bouard [12]. In [12], the author proved that the positive, radially symmetric solitary waves are orbitally stable.

We consider here a generalization of equation (1.2), namely the gBBM-ZK equation:

$$
u_{t}+\varepsilon u_{x}-\left(u_{x t}+\beta u_{y y}\right)_{x}+(f(u))_{x}=0 .
$$

The author in [13] investigated the well-posedness of (1.2). He showed that equation (1.2) is globally well-posed in an appropriate functional space $X$, continuously embedded in $L^{2}\left(\mathbb{R}^{2}\right)$. Furthermore, it was established in [13] that if the solution of the Cauchy problem associated to (1.2) has a compact support for all times, then this solution vanishes identically. It is worth remarking that the transverse instability of solitary waves of (1.2) has been studied in [22].

In the present work, we study the decay behavior of small solutions of the initial value problem for the gBBM-ZK equation. We obtain a lower bound for the degrees of nonlinearity. These results allow us to establish a nonlinear scattering result for small perturbations; that is, the small solutions of the nonlinear problem behave asymptotically like the solution of the associated linear problem.

We also investigate the nonlinear stability of solitary waves of the gBBM-ZK equation. Indeed, by using the famous result of [32], we prove the nonlinear stability and instability of solitary waves of the gBBM-ZK equation under appropriate conditions on the nonlinearity and the parameter $\varepsilon$, and some spectral assumptions. Finally, we will compare the stability of solitary waves of the BBM-ZK equation to the gZK equation and another two dimensional generalization of the BBM equation, the so-called 2D-gBBM equation $[3,12]$ :

$$
u_{t}+\varepsilon u_{x}-\Delta u_{t}+(f(u))_{x}=0 .
$$

Regarding the decay and the scattering of the solutions of (1.7), Biler et al. [6] showed that the supremum norm of solutions with small initial data of (1.7) with $p \geq 3$ decay to zero like $(1+|t|)^{-2 / 3}$. It should be noted that orbital stability of solitary wave solutions and scattering of small solutions to (1.7) were addressed by de Bouard [12].

The rest of this paper is organized as follows. In the next section, the decay and the scattering properties of (1.6) shall be studied. Section 3 is devoted to the nonlinear stability of solitary waves of the gBBM-ZK equation. We will show that solitary waves of the gBBM-ZK equation are stable if and only if $d^{\prime \prime}>0$ (see (3.11)). We will apply our results to the power-law nonlinearity $f(u)=u^{p+1} /(p+1)$. The regions of stability 
of solitary waves of $(1.5),(1.6)$, and (1.7) will be compared. We also study the effects of the parameter $\varepsilon$ on stability of solitary waves of these equations.

We finish this section by introducing some notations which will be used throughout this paper.

Notations. Throughout this paper, the notation $\|\cdot\|_{s, p}$ is used to denote the norm in the Bessel potential space $L_{s}^{p}\left(\mathbb{R}^{n}\right)=(I-\Delta)^{-s / 2} L^{p}\left(\mathbb{R}^{n}\right)$ such that for $u \in$ $L_{s}^{p}\left(\mathbb{R}^{n}\right),\|u\|_{s, p}=\left|(I-\Delta)^{s / 2} u\right|_{p}<+\infty$, where $|\cdot|_{p}$ is denoted the norm in $L^{p}\left(\mathbb{R}^{n}\right)$ space. We also use $\|\cdot\|_{s}$ for the norm in the usual Sobolev space $H^{s}\left(\mathbb{R}^{n}\right)=L_{s}^{2}\left(\mathbb{R}^{n}\right)$. For $1 \leq p \leq \infty$, the notation $|(f, g)|_{p}$ is defined by $|(f, g)|_{p}=|f|_{p}+|g|_{p}$.

We also use the notation $\|\cdot\|_{\left(s_{1}, s_{2}\right)}$ to denote the norm in the anisotropic Sobolev space

$$
H^{s_{1}, s_{2}}\left(\mathbb{R}^{2}\right)=\left(I-\partial_{x}^{2}\right)^{-s_{1} / 2}\left(I-\partial_{y}^{2}\right)^{-s_{2} / 2} L^{2}\left(\mathbb{R}^{2}\right),
$$

such that for $u \in H^{s_{1}, s_{2}}\left(\mathbb{R}^{2}\right)$,

$$
\|u\|_{\left(s_{1}, s_{2}\right)}=\left|\left(I-\partial_{x}^{2}\right)^{s_{1} / 2}\left(I-\partial_{y}^{2}\right)^{s_{2} / 2} u\right|_{2}<+\infty .
$$

For any positive numbers $a$ and $b$, the notation $a \lesssim b$ means that there exists a positive (harmless) constant $k$ such that $a \leq k b$. We also use $a \sim b$ when $a \lesssim b$ and $b \lesssim a$.

\section{Decay and scattering}

In this section, we will study the decay and the scattering properties of (1.6). Throughout this paper we assume that $f \in C^{2}(\mathbb{R})$ such that $f(0)=0$ and $f(s)=$ $O\left(|s|^{p+1}\right)$ as $|s| \rightarrow+\infty$. Before stating our main results, we give the following results for the existence of the local and global solutions of (1.6). The proofs of the first two theorems follow by classical semi-group theory $[28,31]$ or the parabolic regularization theory $[21]$ (see also $[15,32]$ ).

\section{THEOREM 2.1.}

(i) For $u_{0} \in H^{s}\left(\mathbb{R}^{2}\right), s>1$, there exists a $T=T\left(\left\|u_{0}\right\|_{s}\right)>0$, and a unique solution $u \in C\left([0, T) ; H^{s}\left(\mathbb{R}^{2}\right)\right)$ of (1.6) with $u(0)=u_{0}$. In addition, $u(t)$ satisfies $E(u(t))=E\left(u_{0}\right), Q(u(t))=Q\left(u_{0}\right)$, and $I(u(t))=I\left(u_{0}\right)$, for all $t \in[0, T)$, where

$$
\begin{aligned}
I(u) & =\int_{\mathbb{R}^{2}} u \mathrm{~d} x \mathrm{~d} y, \\
E(u) & =-\frac{1}{2} \int_{\mathbb{R}^{2}} \varepsilon u^{2}+\beta u_{y}^{2}+2 F(u) \mathrm{d} x \mathrm{~d} y, \\
Q(u) & =\frac{1}{2} \int_{\mathbb{R}^{2}} u^{2}+u_{x}^{2} \mathrm{~d} x \mathrm{~d} y,
\end{aligned}
$$

where $F^{\prime}=f$. Furthermore,

$$
u \in C\left([0, T) ; H^{s_{1}, s_{2}}\left(\mathbb{R}^{2}\right)\right),
$$

where $s_{1} \geq 0$ and $s_{2}=s-s_{1}$.

(ii) For $u_{0} \in H^{s_{1}, s_{2}}\left(\mathbb{R}^{2}\right), s_{1}, s_{2}>1 / 2$, there exists a $T=T\left(\left\|u_{0}\right\|_{\left(s_{1}, s_{2}\right)}\right)>0$ and a unique solution $u \in C\left([0, T) ; H^{s_{1}, s_{2}}\left(\mathbb{R}^{2}\right)\right)$ of $(1.6)$ with $u(0)=u_{0}$. In addition, $u(t)$ satisfies $E(u(t))=E\left(u_{0}\right), Q(u(t))=Q\left(u_{0}\right)$, and $I(u(t))=I\left(u_{0}\right)$, for all $t \in[0, T)$. Furthermore,

$$
u \in C\left([0, T) ; H^{\min \left\{s_{1}, s_{2}\right\}}\left(\mathbb{R}^{2}\right)\right) .
$$


We also have the following weaker result.

THEOREM 2.2.

(i) If $u_{0} \in H^{1}\left(\mathbb{R}^{2}\right)$, then there exists a global solution $u \in L^{\infty}\left(\mathbb{R} ; H^{1}\left(\mathbb{R}^{2}\right)\right)$ of $(1.6)$ with $u(0)=u_{0}$. Moreover, there exists $T>0$ and a unique weak solution $u \in$ $C\left([0, T) ; H^{1}\left(\mathbb{R}^{2}\right)\right)$ of $(1.6)$ with $u(0)=u_{0}$. In addition, $u(t)$ satisfies $E(u(t))=$ $E\left(u_{0}\right), Q(u(t))=Q\left(u_{0}\right)$, and $I(u(t))=I\left(u_{0}\right)$, for all $t \in[0, T)$.

(ii) If $u_{0} \in H^{1,0}\left(\mathbb{R}^{2}\right)$, then there exists a global solution $u \in L^{\infty}\left(\mathbb{R} ; H^{1,0}\left(\mathbb{R}^{2}\right)\right)$ of $(1.6)$ with $u(0)=u_{0}$. Moreover, there exists $T>0$ and a unique weak solution $u \in$ $C\left([0, T) ; H^{1,0}\left(\mathbb{R}^{2}\right)\right)$ of $(1.6)$ with $u(0)=u_{0}$. In addition, $u(t)$ satisfies $E(u(t))=$ $E\left(u_{0}\right), Q(u(t))=Q\left(u_{0}\right)$, and $I(u(t))=I\left(u_{0}\right)$, for all $t \in[0, T)$.

THEOREM 2.3 .

(i) There is $\delta>0$ such that if $\left\|u_{0}\right\|_{1}<\delta$, equation (1.6) has a unique solution $u \in$ $C\left(\mathbb{R} ; H^{1}\left(\mathbb{R}^{2}\right)\right)$ with $u(0)=u_{0}$. Moreover, $\|u(t)\|_{1} \leq C\left\|u_{0}\right\|_{1}$, for all time $t \in \mathbb{R}$, where $C=C\left(\left\|u_{0}\right\|_{1}\right)>0$ and the functionals $E, Q$, and $I$ are independent of $t$.

(ii) There is $\delta>0$ such that if $\left\|u_{0}\right\|_{(1,0)}<\delta$, equation (1.6) has a unique solution $u \in C\left(\mathbb{R} ; H^{1,0}\left(\mathbb{R}^{2}\right)\right)$ with $u(0)=u_{0}$. Moreover, $\|u(t)\|_{(1,0)} \leq C\left\|u_{0}\right\|_{(1,0)}$, for all time $t \in \mathbb{R}$, where $C=C\left(\left\|u_{0}\right\|_{(1,0)}\right)>0$ and the functionals $E, Q$, and $I$ are independent of $t$.

Proof. Without loss of generality we restrict ourselves to the case $t>0$ and $\beta=-1$. By theorems 2.1 and 2.2 , it suffices to prove that $\|u(t)\|_{1}$ is bounded in $[0, T)$. In fact, using the invariants $E$ and $Q$, we obtain

$$
\|u(t)\|_{1} \leq C\left(\left\|u_{0}\right\|_{1}^{2}+|u(t)|_{p+2}^{p+2}\right) \leq C\left(\left\|u_{0}\right\|_{1}^{2}+\|u(t)\|_{1}^{p+2}\right),
$$

for $t \in[0, T)$, where the constant $C>0$ depends only on $\left\|u_{0}\right\|_{1}$. Define $M(t)=$ $\sup _{0 \leq \tau<t}\|u(\tau)\|_{1}$. Then by (2.4), we have

$$
M(t) \leq C \delta+C M(t)^{q}
$$

where $q=p+2 / 2$. Hence, for sufficiently small $\delta$ such that $\left\|u_{0}\right\|_{1} \leq \delta$, it follows from the continuity of $M(t)$ that $M(t)$ remains in the bounded connected component of $\left\{z \geq 0 z \leq C \delta+C z^{q}\right\}$ containing the origin for all $t \in[0, T)$. Moreover, we have $M(t) \leq$ $C\left\|u_{0}\right\|_{1}$, for all time $t \in[0, T)$. This completes the proof of (i). The proof of (ii) is similar.

Lemma 2.4. Let $N \gg 1, t \neq 0$, and $J=[-N, N]$. Then there exists a constant $C>0$, independent of $t$ and $N$, such that

$$
\left|\int_{J} \int_{\mathbb{R}} \mathrm{e}^{-\mathrm{i}\left(t \xi\left(\frac{\varepsilon+\beta \eta^{2}}{1+\xi^{2}}\right)-x \xi-y \eta\right)} \mathrm{d} \eta \mathrm{d} \xi\right| \leq C t^{-1 / 2} N^{3 / 2} .
$$

Proof. Denote the integral of the left hand side of (2.5) by $A$. Then by the change of variable $\xi=a \sqrt{\frac{1+\xi^{2}}{|t \xi|}}$, we obtain

$$
A=\int_{J} \int_{\mathbb{R}} \mathrm{e}^{-\mathrm{i} \xi\left(\frac{t \xi}{1+\xi^{2}}-x\right)} \mathrm{e}^{-\mathrm{i} \beta a^{2} \operatorname{sgn}(t \xi)+\mathrm{i} y a \sqrt{\frac{1+\xi^{2}}{|t \xi|}}} \sqrt{\frac{1+\xi^{2}}{|t \xi|}} \mathrm{d} a \mathrm{~d} \xi
$$


Now we note that

$$
-\mathrm{i} \beta a^{2} \operatorname{sgn}(t \xi)+\mathrm{i} y a \sqrt{\frac{1+\xi^{2}}{|t \xi|}}=-\mathrm{i} \beta \operatorname{sgn}(t \xi)\left(a-\frac{\beta y \operatorname{sgn}(t \xi)}{2} \sqrt{\frac{1+\xi^{2}}{|t \xi|}}\right)^{2}+\frac{\mathrm{i} \beta y^{2}\left(1+\xi^{2}\right)}{4|t \xi|},
$$

hence

$$
\int_{\mathbb{R}} \mathrm{e}^{-\mathrm{i} \beta a^{2} \operatorname{sgn}(t \xi)+\mathrm{i} y a \sqrt{\frac{1+\xi^{2}}{|t \xi|}}} \mathrm{d} a=\mathrm{e}^{\frac{\mathrm{i} \beta y^{2}\left(1+\xi^{2}\right)}{4|t \xi|}} \int_{\mathbb{R}} \mathrm{e}^{ \pm a^{2}} \mathrm{~d} a=\sqrt{\pi} \mathrm{e}^{\frac{\mathrm{i} \beta y^{2}\left(1+\xi^{2}\right)}{4|t \xi|} \pm \mathrm{i} \frac{\pi}{4}} .
$$

Consequently, this yields that

$$
|A|=\sqrt{\pi}\left|t^{-1 / 2}\right|\left|\int_{J} \sqrt{\frac{1+\xi^{2}}{|\xi|}} \mathrm{d} \xi\right| \lesssim t^{-1 / 2} N^{3 / 2}
$$

LEMMA 2.5. Let $S(t)$ be the $C_{0}$-group of unitary operators for the linearized problem of (1.6),

$$
u_{t}+\varepsilon u_{x}-\left(u_{x t}+\beta u_{y y}\right)_{x}=0 .
$$

(i) Let $s_{1}, s_{2}>1 / 2, \theta=\left(2\left(s_{1}+1\right)\right)^{-1} \in[0,1 / 3)$, and $u_{0} \in H^{s_{1}, s_{2}}(\mathbb{R}) \cap L^{1}\left(\mathbb{R}^{2}\right)$. Then there exists a constant $C>0$, depending only on $s_{1}$, such that for all $t \in \mathbb{R}$ we have

$$
\left|S(t) u_{0}\right|_{\infty} \leq C(1+|t|)^{\frac{3 \theta-1}{2}}\left(\left|u_{0}\right|_{1}+\left\|u_{0}\right\|_{\left(s_{1}, s_{2}\right)}\right) .
$$

(ii) Let $s>1 / 2, \theta=(2(s+1))^{-1} \in[0,1 / 3)$, and $u_{0} \in H^{2 s}(\mathbb{R}) \cap L^{1}\left(\mathbb{R}^{2}\right)$. Then there exists a constant $C>0$, depending only on $s$, such that for all $t \in \mathbb{R}$ we have

$$
\left|S(t) u_{0}\right|_{\infty} \leq C(1+|t|)^{\frac{3 \theta-1}{2}}\left(\left|u_{0}\right|_{1}+\left\|u_{0}\right\|_{2 s}\right) .
$$

Proof. (i) Let $N>0$ be sufficiently large. Then we have

$$
\begin{aligned}
S(t) u_{0}=\int_{|\xi|>N} & \int_{\mathbb{R}} \mathrm{e}^{\mathrm{i}(t h(\xi, \eta)+x \xi+y \eta)} \widehat{u}_{0}(\xi, \eta) \mathrm{d} \eta \mathrm{d} \xi \\
& +\int_{|\xi| \leq N} \int_{\mathbb{R}} \mathrm{e}^{\mathrm{i}(t h(\xi, \eta)+x \xi+y \eta)} \widehat{u}_{0}(\xi, \eta) \mathrm{d} \eta \mathrm{d} \xi
\end{aligned}
$$

where $h(\xi, \eta)=\xi\left(\frac{\varepsilon+\beta \eta^{2}}{1+\xi^{2}}\right)$. By Lemma 2.4, the second term in the right hand side of the above identity is bounded by $t^{-1 / 2} N^{3 / 2}\left|u_{0}\right|_{1}$. On the other hand, the CauchySchwarz inequality implies that

$$
\left|\int_{|\xi| \geq N} \int_{\mathbb{R}} \mathrm{e}^{\mathrm{i}(t h(\xi, \eta)+x \xi+y \eta)} \widehat{u}_{0}(\xi, \eta) \mathrm{d} \eta \mathrm{d} \xi\right| \lesssim N^{1 / 2-s_{1}}\left\|u_{0}\right\|_{\left(s_{1}, s_{2}\right)} .
$$

By choosing $N=t^{\theta}$ with $|t| \geq 1$, we obtain

$$
\left|S(t) u_{0}\right| \lesssim|t|^{\frac{3 \theta-1}{2}}\left(\left|u_{0}\right|_{1}+\left\|u_{0}\right\|_{\left(s_{1}, s_{2}\right)}\right),
$$

for all $|t| \geq 1$. Finally by using the embedding $H^{s_{1}, s_{2}}\left(\mathbb{R}^{2}\right) \hookrightarrow L^{\infty}\left(\mathbb{R}^{2}\right)$ for $s_{1}, s_{2}>1 / 2$, we have for $|t| \leq 1$ that

$$
\left|S(t) u_{0}\right| \lesssim\left\|u_{0}\right\|_{\left(s_{1}, s_{2}\right)}
$$


This completes the proof of (i).

The proof of (ii) is similar to (i).

The following lemma is a direct consequence of Lemma 2.5 and an interpolation theorem.

Lemma 2.6. Let $1<q \leq \infty$ and $1 / q+1 / q^{\prime}=1$.

(i) If $s_{1}, s_{2}>1 / 2, \quad \theta=\left(2\left(s_{1}+1\right)\right)^{-1} \in[0,1 / 3)$, and $u_{0} \in H^{s_{1}, s_{2}}(\mathbb{R}) \cap L^{q}\left(\mathbb{R}^{2}\right)$, then there exists a constant $C>0$, depending only on $s_{1}$, such that for all $t \in \mathbb{R}$ we have

$$
\left|S(t) u_{0}\right|_{q^{\prime}} \leq C(1+|t|)^{\left(\frac{3 \theta-1}{2}\right)\left(1-\frac{2}{q^{\prime}}\right)}\left(\left|u_{0}\right|_{q}+\left\|u_{0}\right\|_{\left(s_{1}, s_{2}\right)}\right) .
$$

(ii) If $s>1 / 2, \theta=(2(s+1))^{-1} \in[0,1 / 3)$, and $u_{0} \in H^{2 s}\left(\mathbb{R}^{2}\right) \cap L^{q}\left(\mathbb{R}^{2}\right)$, then there exists a constant $C>0$, depending only on $s$, such that for all $t \in \mathbb{R}$ we have

$$
\left|S(t) u_{0}\right|_{q^{\prime}} \leq C(1+|t|)^{\left(\frac{3 \theta-1}{2}\right)\left(1-\frac{2}{q^{\prime}}\right)}\left(\left|u_{0}\right|_{q}+\left\|u_{0}\right\|_{2 s}\right) .
$$

Theorem 2.7. Suppose that $s>1, \mu=(1-3 \theta) / 2, \theta=(2(s+1))^{-1} \in[0,1 / 3)$, and

$$
p>\frac{3(1-\theta)}{1-3 \theta} \text {. }
$$

Then there exists $\delta>0$ and $C>0$ such that for any $u_{0} \in H^{2 s}\left(\mathbb{R}^{2}\right) \cap L^{1}\left(\mathbb{R}^{2}\right)$ satisfying $\left|u_{0}\right|_{1}+\left\|u_{0}\right\|_{2 s}<\delta$, there is a unique solution $u \in C\left(\mathbb{R} ; H^{2 s}\left(\mathbb{R}^{2}\right)\right)$ of $(1.6)$ with $u(0)=u_{0}$ such that

$$
|u(t)|_{\infty} \leq C(1+|t|)^{-\mu}
$$

Proof. Consider the map

$$
\Phi(u)(t)=S(t) u_{0}+\int_{0}^{t} S(t-\tau) \mathcal{L}^{-1} \partial_{x} f(u(\tau)) \mathrm{d} \tau,
$$

where $\mathcal{L}=I-\partial_{x}^{2}$, and for $T>0$ define the norm $\|u\|_{\mathscr{X}}$, for $u \in H^{2 s}\left(\mathbb{R}^{2}\right)$, by

$$
\|u\|_{\mathscr{X}}:=\sup _{t \in[0, T]}\left(\|u(\tau)\|_{2 s}+(1+|\tau|)^{\mu}|u(\tau)|_{\infty}\right) .
$$

We shall show that $\Phi$ in strictly contractive in a suitable closed ball in $C\left([-T, T] ; H^{2}\left(\mathbb{R}^{2}\right)\right)$. First we prove that

$$
|\Phi(u)-\Phi(v)|_{\infty}(t) \leq C_{s, p}\left(\|u\|_{\mathscr{X}}^{p}+\|v\|_{\mathscr{X}}^{p}\right)(1+|t|)^{-\mu}\|u-v\|_{\mathscr{X}},
$$

for any $u, v \in H^{2 s}\left(\mathbb{R}^{2}\right)$. Indeed, for any $u, v \in H^{2 s}\left(\mathbb{R}^{2}\right)$, we have from (2.11) and Lemma 2.5 that

$$
\begin{aligned}
|\Phi(u)-\Phi(v)|_{\infty}(t) & \lesssim \int_{0}^{t}\left|S(t-\tau) \mathcal{L}^{-1} \partial_{x}(F(u(\tau))-F(v(\tau)))\right|_{\infty} \mathrm{d} \tau \\
& \lesssim \int_{0}^{t}(1+t-\tau)^{-\mu}\left(\left|\mathcal{L}^{-1} \partial_{x}(F(u(\tau))-F(v(\tau)))\right|_{1}\right. \\
& \left.+\left\|\mathcal{L}^{-1} \partial_{x}(F(u(\tau))-F(v(\tau)))\right\|_{2 s}\right) \mathrm{d} \tau \\
& \lesssim \int_{0}^{t}(1+t-\tau)^{-\mu}\left(|F(u(\tau))-F(v(\tau))|_{1}\right. \\
& \left.+\|F(u(\tau))-F(v(\tau))\|_{2 s}\right) \mathrm{d} \tau
\end{aligned}
$$


where in the last inequality we used the fact

$$
\begin{aligned}
\left|\mathcal{L}^{-1} \partial_{x} F\right|_{1} & \left.\left.\left.\left.\lesssim|| \mathcal{L}^{-1} \partial_{x} F\right|_{L_{x}^{1}(\mathbb{R})}\right|_{L_{y}^{1}(\mathbb{R})} \lesssim|| K\right|_{L_{x}^{1}(\mathbb{R})}|F|_{L_{x}^{1}(\mathbb{R})}\right|_{L_{y}^{1}(\mathbb{R})} \\
& =\left.\left.|K|_{L_{x}^{1}(\mathbb{R})}|| F\right|_{L_{x}^{1}(\mathbb{R})}\right|_{L_{y}^{1}(\mathbb{R})}=|K|_{L_{x}^{1}(\mathbb{R})}|F|_{1},
\end{aligned}
$$

where $K(x)=\operatorname{sgn}(x) \mathrm{e}^{-|x|} \in L^{1}(\mathbb{R})$. Now, we are going to estimate $\| F(u(\tau))-$ $F(v(\tau)) \|_{1}$ and $\|F(u(\tau))-F(v(\tau))\|_{2 s}$. Actually, for the $H^{2 s}\left(\mathbb{R}^{2}\right)$-estimate, by using the fractional Leibniz rule [23, Lemma X.4] we have

$$
\begin{aligned}
\|F(u(t))-F(v(t))\|_{2 s} & \lesssim|u-v|_{\infty}\left\||u|^{p}+|v|^{p}\right\|_{2 s}+\left\|\left.u\right|^{p}+\left.|v|^{p}\right|_{\infty}\right\| u-v \|_{2 s} \\
& \lesssim|u-v|_{\infty}\|u|+| v\|_{\infty}^{p-1}\||u|+|v|\|_{2 s}+\left\|\left.u\right|^{p}+\left.|v|^{p}\right|_{\infty}\right\| u-v \|_{2 s} \\
& \lesssim(1+\tau)^{-\mu p}\left(\|u\|_{\mathscr{X}}^{p}+\|v\|_{\mathscr{X}}^{p}\right)\|u-v\|_{\mathscr{X}} .
\end{aligned}
$$

On the other hand, it follows from the Cauchy-Schwarz inequality that

$$
\begin{aligned}
|F(u(t))-F(v(t))|_{1} & \left.\lesssim|u-v|_{\infty}|| u\right|^{p-2}+\left.|v|^{p-2}\right|_{\infty}|| u|+| v \|_{2}^{2} \\
& \left.\lesssim|u-v|_{\infty}|| u\right|^{p-2}+\left.|v|^{p-2}\right|_{\infty}\||u|+|v|\|_{2 s}^{2} \\
& \lesssim(1+\tau)^{-\mu(p-1)}\left(\|u\|_{\mathscr{X}}^{p}+\|v\|_{\mathscr{X}}^{p}\right)\|u-v\|_{\mathscr{X}} .
\end{aligned}
$$

We therefore conclude that

$$
|\Phi(u(t))-\Phi(v(t))|_{\infty} \lesssim(1+t)^{-\mu(p-1)}\left(\|u\|_{\mathscr{X}}^{p}+\|v\|_{\mathscr{X}}^{p}\right)\|u-v\|_{\mathscr{X}} .
$$

Next, we show that

$$
\|\Phi(u)(t)-\Phi(v)(t)\|_{2 s} \leq C_{s, p}\left(\|u\|_{\mathscr{X}}^{p}+\|v\|_{\mathscr{X}}^{p}\right)(1+|t|)^{-\mu}\|u-v\|_{\mathscr{X}},
$$

for any $u, v \in H^{2 s}\left(\mathbb{R}^{2}\right)$. But, for any $u, v \in H^{2 s}\left(\mathbb{R}^{2}\right)$, we have from (2.11) and the Leibniz rule that

$$
\begin{aligned}
\|\Phi(u)(t)-\Phi(v)(t)\|_{2 s} & \lesssim \int_{0}^{t}\|F(u(\tau))-F(v(\tau))\|_{2 s} \mathrm{~d} \tau \\
& \lesssim \int_{0}^{t}|u-v|_{\infty}\left\||u|^{p}+|v|^{p}\right\|_{2 s}+\|u-v\|_{2 s}\|u|+| v\|_{\infty}^{p} \mathrm{~d} \tau \\
& \lesssim \int_{0}^{t}|u-v|_{\infty}|| u|+| v\left\|_{\infty}^{p-1}\right\||u|+|v|\left\|_{2 s}+\right\| u-v\left\|_{2 s}\right\| u|+| v \|_{\infty}^{p} \mathrm{~d} \tau \\
& \lesssim\left(\|u\|_{\mathscr{X}}^{p}+\|v\|_{\mathscr{X}}^{p}\right)\|u-v\|_{\mathscr{X}} \int_{0}^{t}(1+\tau)^{-\mu p} \mathrm{~d} \tau \\
& \lesssim\left(\|u\|_{\mathscr{X}}^{p}+\|v\|_{\mathscr{C}}^{p}\right)\|u-v\|_{\mathscr{X}} .
\end{aligned}
$$

Combining (2.12) and (2.14) leads to the conclusion

$$
\|\Phi(u)-\Phi(v)\|_{\mathscr{X}} \leq C_{s, p}\left(\|u\|_{\mathscr{X}}^{p}+\|v\|_{\mathscr{X}}^{p}\right)\|u-v\|_{\mathscr{X}}
$$

and

$$
\|\Phi(u)\|_{\mathscr{X}} \leq C_{s, p}\left|u_{0}\right|+\left\|u_{0}\right\|_{2 s}+\|u\|_{\mathscr{X}}^{p+1}
$$

Now for $M>0$, we consider the closed ball

$$
B_{\mathscr{X}}=\left\{u \in C\left([-T, T] ; H^{2 s}\left(\mathbb{R}^{2}\right)\right) ;\|u\|_{\mathscr{X}} \leq M\right\} .
$$


By choosing $\delta>0$ and $M>0$ small enough such that $\delta+M^{p+1} \leq M$ and $C_{s, p} M<1$, it follows from (2.15) and (2.16) that $\Phi$ is strictly contractive on $B_{\mathscr{X}}$ and there exists a unique solution $u$ of (1.6) in $B \mathscr{X}$, if $\left|u_{0}\right|_{1}+\left\|u_{0}\right\|<\delta$. Thereby, the continuity of the norm $\|u\|_{\mathscr{X}}$ with respect to $T$ and (2.16) allows us to conclude that $\|u\|_{\mathscr{X}}$ remains bounded for all $T>0$. Thus we have obtained a bound of $\|u\|_{\mathscr{X}}$ for all $T$ and consequently an a priori estimate of the local solution which permits us to extend $u$ globally.

Theorem 2.8. Let $r, s>1, q^{\prime}>2, s^{\prime}>2 / r, \mu=(1-3 \theta) / 2<q^{\prime} /\left(q^{\prime}-2\right), \theta=(2(s+$ $1))^{-1} \in[0,1 / 3)$, and

$$
p>\frac{4\left(r-q^{\prime}\right)+2 r q^{\prime}}{(1-3 \theta)\left(q^{\prime}-2\right)(r s-2)}
$$

Then there exists $\delta>0$ and $C>0$ such that for any $u_{0} \in H^{2 s}\left(\mathbb{R}^{2}\right) \cap L^{q^{\prime}}\left(\mathbb{R}^{2}\right) \cap L_{s^{\prime}}^{r}\left(\mathbb{R}^{2}\right)$ satisfying $\left|u_{0}\right|_{q^{\prime}}+\left\|u_{0}\right\|_{2 s}+\left\|u_{0}\right\|_{s^{\prime}, r}<\delta$, there is a unique solution $u \in C\left(\mathbb{R} ; H^{2 s}\left(\mathbb{R}^{2}\right) \cap\right.$ $\left.L_{s^{\prime}}^{r}\left(\mathbb{R}^{2}\right)\right)$ of $(1.6)$ with $u(0)=u_{0}$ such that

$$
|u(t)|_{q^{\prime}} \leq C(1+|t|)^{-\mu\left(1-\frac{2}{q^{\prime}}\right)} .
$$

Proof. Let $\ell=1-2 / q^{\prime}$. For $T>0$, define the norm $\|u\|_{\mathscr{X}}$, for $u \in H^{2 s}\left(\mathbb{R}^{2}\right) \cap$ $L_{s^{\prime}}^{r}\left(\mathbb{R}^{2}\right)$, by

$$
\|u\|_{\mathscr{X}}:=\sup _{0 \leq \tau \leq T}\left(\|u(\tau)\|_{2 s}+\|u(\tau)\|_{s^{\prime}, r}+(1+\tau)^{\mu \ell}|u(\tau)|_{q^{\prime}}\right) .
$$

Similar to Theorem 2.7, it is enough to show that $\Phi$, defined in (2.11), in strictly contractive in a suitable closed ball in $C\left([-T, T] ; H^{2 s}\left(\mathbb{R}^{2}\right) \cap L_{s^{\prime}}^{r}\left(\mathbb{R}^{2}\right)\right)$; we therefore show that, for any $u, v \in H^{2 s}\left(\mathbb{R}^{2}\right) \cap L_{s^{\prime}}^{r}\left(\mathbb{R}^{2}\right)$,

$$
\begin{gathered}
|\Phi(u)-\Phi(v)|_{q^{\prime}} \leq C\left(\|u\|_{\mathscr{X}}^{p}+\|v\|_{\mathscr{X}}^{p}\right)(1+t)^{-\mu \ell}\|u-v\|_{\mathscr{X}}, \\
\|\Phi(u)-\Phi(v)\|_{s^{\prime}, r} \leq C\left(\|u\|_{\mathscr{X}}^{p}+\|v\|_{\mathscr{X}}^{p}\right)\|u-v\|_{\mathscr{X}}, \\
\|\Phi(u)-\Phi(v)\|_{2 s} \leq C\left(\|u\|_{\mathscr{X}}^{p}+\|v\|_{\mathscr{X}}^{p}\right)\|u-v\|_{\mathscr{X}},
\end{gathered}
$$

where $C=C\left(s, p, s^{\prime}, r\right)$. To prove (2.18), note that (2.11), Lemma 2.6, and (2.13) imply that

$$
\begin{aligned}
|\Phi(u)-\Phi(v)|_{q^{\prime}} & \lesssim \int_{0}^{t}\left|S(t-\tau) \mathcal{L}^{-1} \partial_{x}(F(u(\tau))-F(v(\tau)))\right|_{q^{\prime}} \mathrm{d} \tau \\
& \lesssim \int_{0}^{t}(1+t-\tau)^{-\mu}\left(\left|\mathcal{L}^{-1} \partial_{x}(F(u(\tau))-F(v(\tau)))\right|_{q}\right. \\
& \left.\quad+\left\|\mathcal{L}^{-1} \partial_{x}(F(u(\tau))-F(v(\tau)))\right\|_{2 s}\right) \mathrm{d} \tau \\
& \lesssim \int_{0}^{t}(1+t-\tau)^{-\mu}\left(|F(u(\tau))-F(v(\tau))|_{q}+\|F(u(\tau))-F(v(\tau))\|_{2 s}\right) \mathrm{d} \tau
\end{aligned}
$$

where in the last inequality we used the Mikhlin-Hormander theorem [33]. It remains to bound $F(u(\tau))-F(v(\tau))$ in $H^{2 s}\left(\mathbb{R}^{2}\right) \cap L^{q}\left(\mathbb{R}^{2}\right)$. First, observe from the Hölder 
inequality, the Sobolev embedding, and the Gagliardo-Nirenberg inequality [18] that

$$
\begin{aligned}
|F(u(\tau))-F(v(\tau))|_{q} & \left.\lesssim|u-v|_{q^{\prime}}|| u\right|^{p}+\left.|v|^{p}\right|_{\frac{q q^{\prime}}{q^{\prime}-q}} \\
& \left.\lesssim|u-v|_{q^{\prime}}|| u|+| v\right|_{\frac{p q q^{\prime}}{q^{\prime}-q}} ^{p} \lesssim|u-v|_{q^{\prime}}\||u|+|v|\|_{s^{\prime}, r}^{p} \\
& \lesssim|u-v|_{q^{\prime}}\left|\left\|u|+| v\left|\|_{s^{\prime}, r}\right||u|+|v|_{\infty}^{p-1}\right.\right. \\
& \lesssim|u-v|_{q^{\prime}}\||u|+|v|\|_{s^{\prime}, r}\||u|+|v|\|_{s^{\prime}, r}^{a(p-1)}\|u|+| v\|_{q^{\prime}}^{(1-a)(p-1)} \\
& \lesssim(1+\tau)^{-\mu \ell(1-a)}\|u-v\|_{\mathscr{X}}\left(\|u\|_{\mathscr{X}}+\|v\|_{\mathscr{X}}\right)^{p}
\end{aligned}
$$

where

$$
a=\frac{2 r}{2\left(r-q^{\prime}\right)+r q^{\prime}}
$$

On the other hand, it is deduced from the Leibniz rule and the Gagliardo-Nirenberg inequality that

$$
\begin{aligned}
& \|F(u(\tau))-F(v(\tau))\|_{2 s} \\
\lesssim & \left.\|u-v\|_{2 s}|| u\right|^{p}+\left.|v|^{p}\right|_{\infty}+|u-v|_{\infty}\left\||u|^{p}+|v|^{p}\right\|_{2 s} \\
\lesssim & \|u-v\|_{2 s}\||u|+|v|\|_{s^{\prime}, r}^{a p}|| u|+| v\left\|_{q^{\prime}}^{(1-a) p}+\right\| u-v\left\|_{s^{\prime}, r}^{a}|u-v|_{q^{\prime}}^{1-a}\right\||u|^{p}+|v|^{p} \|_{2 s} \\
\lesssim & \|u-v\|_{2 s}\||u|+|v|\|_{s^{\prime}, r}^{a p}|| u|+| v \|_{q^{\prime}}^{(1-a) p} \\
& \quad+\|u-v\|_{s^{\prime}, r}^{a}|u-v|_{q^{\prime}}^{1-a}\||u|+|v|\|_{2 s}\|u|+| v\|_{\infty}^{p-1} \\
\lesssim & \|u-v\|_{2 s}\||u|+|v|\|_{s^{\prime}, r}^{a p}|| u|+| v \|_{q^{\prime}}^{(1-a) p} \\
& \quad+\|u-v\|_{s^{\prime}, r}^{a}|u-v|_{q^{\prime}}^{1-a}\||u|+|v|\|_{2 s}\||u|+|v|\|_{s^{\prime}, r}^{a(p-1)}\|u|+| v\|_{q^{\prime}}^{(1-a)(p-1)} \\
\lesssim & (1+\tau)^{-\mu \ell(1-a)}\|u-v\|_{\mathscr{X}}\left(\|u\|_{\mathscr{X}}+\|v\|_{\mathscr{X}}\right)^{p},
\end{aligned}
$$

where $a$ is the same as above. It is then concluded that

$$
\begin{aligned}
|\Phi(u)-\Phi(v)|_{q^{\prime}} & \lesssim\|u-v\|_{\mathscr{X}}\left(\|u\|_{\mathscr{X}}+\|v\|_{\mathscr{X}}\right)^{p} \int_{0}^{t}(1+\tau)^{-\mu \ell(1-a)}(1+t-\tau)^{-\mu} \mathrm{d} \tau \\
& \lesssim(1+t)^{-\mu}\|u-v\|_{\mathscr{X}}\left(\|u\|_{\mathscr{X}}+\|v\|_{\mathscr{X}}\right)^{p}
\end{aligned}
$$

To verify (2.19), one can observe again from (2.11) that

$$
\begin{aligned}
\|\Phi(u)-\Phi(v)\|_{s^{\prime}, r} & \lesssim \int_{0}^{t}\left\|\mathcal{L}^{-1} \partial_{x}(F(u(\tau))-F(v(\tau)))\right\|_{s^{\prime}, r} \mathrm{~d} \tau \\
& \lesssim \int_{0}^{t}\left\|\mathcal{L}^{-1} \partial_{x}(F(u(\tau))-F(v(\tau)))\right\|_{s^{\prime}, r} \mathrm{~d} \tau
\end{aligned}
$$


But we get from the Leibniz rule and the Gagliardo-Nirenberg inequality that

$$
\begin{aligned}
& \|F(u(\tau))-F(v(\tau))\|_{s^{\prime}, r} \\
\lesssim & \left.\|u-v\|_{s^{\prime}, r}\left\|\left.u\right|^{p}+\left.|v|^{p}\right|_{\infty}+|u-v|_{\infty}\right\||| u\right|^{p}+|v|^{p} \|_{s^{\prime}, r} \\
\lesssim & \|u-v\|_{s^{\prime}, r}\||u|+|v|\|_{s^{\prime}, r}^{a p}|| u|+| v \|_{q^{\prime}}^{(1-a) p} \\
& \quad+\|u-v\|_{s^{\prime}, r}^{a}|u-v|_{q^{\prime}}^{1-a}\left\||u|^{p}+|v|^{p}\right\|_{s^{\prime}, r} \\
\lesssim & \|u-v\|_{s^{\prime}, r}\||u|+|v|\|_{s^{\prime}, r}^{a p}|| u|+| v \|_{q^{\prime}}^{(1-a) p} \\
& \quad+\|u-v\|_{s^{\prime}, r}^{a}|u-v|_{q^{\prime}}^{1-a}\||u|+|v|\|_{s^{\prime}, r}\|u|+| v\|_{\infty}^{p-1} \\
\lesssim & \|u-v\|_{s^{\prime}, r}\||u|+|v|\|_{s^{\prime}, r}^{a p}|| u|+| v \|_{q^{\prime}}^{(1-a) p} \\
& \quad+\|u-v\|_{s^{\prime}, r}^{a}|u-v|_{q^{\prime}}^{1-a}\||u|+|v|\|_{s^{\prime}, r}\|u|+| v\|_{q^{\prime}}^{(1-a)(p-1)}\||u|+|v|\|_{s^{\prime}, r}^{a(p-1)} \\
\lesssim & (1+\tau)^{-\mu \ell(1-a)}\|u-v\|_{\mathscr{X}}\left(\|u\|_{\mathscr{X}}+\|v\|_{\mathscr{X}}\right)^{p},
\end{aligned}
$$

where $a$ is defined in (2.21). It is therefore concluded that

$$
\begin{aligned}
\|\Phi(u)-\Phi(v)\|_{s^{\prime}, r} & \lesssim\|u-v\|_{\mathscr{X}}\left(\|u\|_{\mathscr{X}}+\|v\|_{\mathscr{X}}\right)^{p} \int_{0}^{t}(1+\tau)^{-\mu \ell(1-a)} \mathrm{d} \tau \\
& \lesssim\|u-v\|_{\mathscr{X}}\left(\|u\|_{\mathscr{X}}+\|v\|_{\mathscr{X}}\right)^{p} .
\end{aligned}
$$

Inequality (2.20) can be proved similar to (2.18) and (2.19).

We proceed by studying the asymptotic behavior of such solutions as $t \rightarrow \pm \infty$. We prove that under a smallness condition the solution scatters to a solution of the linear problem. Precisely, we have the following.

THEOREM 2.9. Under assumptions of Theorem 2.7, let $u(t)$ be the solution of (1.6). Then there exists a unique solution $u^{ \pm}(t) \in H^{2 s}\left(\mathbb{R}^{2}\right), s \geq 1 / 2$, of the linearized equation of (1.6) such that

$$
\left\|u(t)-u^{ \pm}(t)\right\|_{2 s} \rightarrow 0
$$

as $t \rightarrow \pm \infty$.

Proof. Let $u(t)$ be the solution of (1.6) in Theorem 2.7. We define

$$
u^{ \pm}(t)=u(t)+\int_{t}^{ \pm \infty} S(t-\tau) \mathcal{L}^{-1} \partial_{x} f(u(\tau)) \mathrm{d} \tau,
$$

which is a solution of the linearized equation of (1.6):

$$
u_{t}+\varepsilon u_{x}-\left(u_{x t}+\beta u_{y y}\right)_{x}=0 .
$$

Now we only consider the case $u^{+}(t)$, since the proof of $u^{-}(t)$ is similar.

By theorems 2.3 and 2.7, we obtain

$$
\begin{aligned}
\left\|u(t)-u^{+}(t)\right\|_{2 s} & \leq \int_{t}^{+\infty}\left\|S(t-\tau) \mathcal{L}^{-1} \partial_{x} f(u(\tau))\right\|_{2 s} \mathrm{~d} \tau \\
& \leq \int_{t}^{+\infty}\|f(u(\tau))\| \mathrm{d} \tau \lesssim \int_{t}^{+\infty}|u|_{\infty}^{p}\|u\|_{2 s} \mathrm{~d} \tau \\
& \leq C \delta \int_{t}^{+\infty}(1+\tau)^{-p \mu} \mathrm{d} \tau \leq C \delta(1+t)^{1-p \mu} \rightarrow 0,
\end{aligned}
$$


as $t \rightarrow+\infty$. Using the integral form (2.11), we may write $u^{+}(t)$ as the form

$$
u^{+}(t)=S(t) u_{0}+\int_{0}^{+\infty} S(t-\tau) \mathcal{L}^{-1} \partial_{x} f(u(\tau)) \mathrm{d} \tau=S(t) w^{+},
$$

where $w^{+}=u_{0}+\int_{0}^{+\infty} S(-\tau) \mathcal{L}^{-1} \partial_{x} f(u(\tau)) \mathrm{d} \tau$. Since the right side is a linear combination of solutions of (2.24), so is $u^{+}$. Now we are going to show the uniqueness of $u^{+}$. Let $w^{+}$be another solution of (2.24) which satisfies (2.22). Let $V(t)=u^{+}(t)-w^{+}(t)$. We want to show that $V(t)=0$ a.e. Since $V$ is also the solution of $(2.24)$, we have

$$
\|V(0)\|_{2 s}=\|S(t) V(0)\|_{2 s}=\|V(t)\|_{2 s}\left\|u(t)-w^{+}(t)\right\|_{2 s}+\left\|u(t)-u^{+}(t)\right\|_{2 s} \rightarrow 0,
$$

as $t \rightarrow+\infty$. This implies that $\|V(t)\|_{1}=0$ for all $t \geq 0$. Hence $u^{+}=w^{+}$. This completes the proof of Theorem 2.9 .

The following theorem plays a key role in our instability analysis.

TheOREM 2.10. Let $u_{0} \in H^{s_{1}, s_{2}}\left(\mathbb{R}^{2}\right) \cap L^{\infty}\left(\mathbb{R}_{y} ; L^{1}\left(\mathbb{R}_{x}\right)\right) \cap L^{1}\left(\mathbb{R}^{2}\right), s_{1}, s_{2}>1 / 2$. Then if $u(t)$ is the solution of (1.6) with $u(0)=u_{0}$, we have

$$
\left|\partial_{x}^{-1} u(z, y, t)\right|_{\infty} \leq C(1+|t|)^{\frac{3 \theta+1}{2}},
$$

where $\theta=\left(2\left(s_{1}+1\right)\right)^{-1} \in(0,1 / 3)$ and $C$ is a constant depending only on

$$
\sup _{t \geq 0}\|u(t)\|_{1}+\left\|u_{0}\right\|_{L_{y}^{\infty} L_{x}^{1}}+\left|u_{0}\right|_{1} .
$$

Proof. Considering the group $S(t)$ defined above, we can write

$$
u(t)=S(t) u_{0}-\int_{0}^{t} S(t-\tau) \mathcal{L}^{-1} \partial_{x} f(u(\tau)) \mathrm{d} \tau,
$$

so that

$$
\begin{aligned}
U(t) & =\partial_{x}^{-1} u(t)=\int_{-\infty}^{x} u(z, y, t) \mathrm{d} z=Z(t)-\int_{0}^{t} S(t-\tau) \mathcal{L}^{-1} f(u(\tau)) \mathrm{d} \tau, \\
& =Z(t)-\Lambda(t),
\end{aligned}
$$

where $Z(t)=\partial_{x}^{-1} S(t) u_{0}$. On the other hand, we can write

$$
S(t) u_{0}=u_{0}-\int_{0}^{t} \mathcal{L}^{-1} \partial_{x} S(\tau) u_{0} \mathrm{~d} \tau
$$

Then, we have

$$
Z(t)=U_{0}-\int_{0}^{t} \mathcal{L}^{-1} S(\tau) u_{0} \mathrm{~d} \tau,
$$

where $U_{0}=\partial_{x}^{-1} u_{0}$. Now by our assumptions we get from Lemma 2.5 that

$$
\begin{aligned}
|Z(t)| & \leq\left|\int_{-\infty}^{x} u_{0}(z, y) \mathrm{d} z\right|+\left|\int_{0}^{t} \mathcal{L}^{-1} S(\tau) u_{0} \mathrm{~d} \tau\right| \\
& \leq\left|\int_{\mathbb{R}} u_{0}(z, y) \mathrm{d} z\right|+\int_{0}^{t}\left|\mathcal{L}^{-1} S(\tau) u_{0}\right|_{\infty} \mathrm{d} \tau \\
& \leq\left|u_{0}\right|_{L^{\infty}\left(\mathbb{R}_{y} ; L^{1}\left(\mathbb{R}_{x}\right)\right)}+\int_{0}^{t}\left|S(\tau) \mathcal{L}^{-1} u_{0}\right|_{\infty} \mathrm{d} \tau \\
& \lesssim\left|u_{0}\right|_{L^{\infty}\left(\mathbb{R}_{y} ; L^{1}\left(\mathbb{R}_{x}\right)\right)}+(1+|t|)^{\frac{3 \theta+1}{2}}\left(\left|u_{0}\right|_{1}+\left\|u_{0}\right\|_{\left(s_{1}, s_{2}\right)}\right),
\end{aligned}
$$


where in the last inequality we used the fact $\left|\mathcal{L}^{-1} u_{0}\right|_{1} \lesssim\left|u_{0}\right|_{1}$ (see (2.13)). It just remains to estimate $\Lambda(t)$. Note that another application of Lemma 2.5 yields

$$
\begin{aligned}
|\Lambda(t)| & \lesssim \int_{0}^{t}(1+t-\tau)^{\frac{3 \theta-1}{2}}\left(\left|\mathcal{L}^{-1} f(u)\right|_{1}+\left\|\mathcal{L}^{-1} f(u)\right\|_{\left(s_{1}, s_{2}\right)}\right) \mathrm{d} \tau \\
& \lesssim \int_{0}^{t}(1+t-\tau)^{\frac{3 \theta-1}{2}}\left(|f(u)|_{1}+|f(u)|_{2}\right) \mathrm{d} \tau .
\end{aligned}
$$

Since $|f(s)| \leq C|s|^{2}$ for $|s| \leq 1$ and $|f(s)| \leq C|s|^{p+1}$ for $|s| \geq 1$,

$|f(u)|_{1} \leq C\left(\int_{|u| \leq 1}|u|^{2} \mathrm{~d} x \mathrm{~d} y+\int_{|u| \geq 1}|u|^{p+1} \mathrm{~d} x \mathrm{~d} y\right) \leq C\left(|u|_{2}^{2}+|u|_{p+1}^{p+1}\right) \leq C=C\left(\|u\|_{1}\right)$.

It is therefore concluded that $|\Lambda(t)| \leq C(1+t)^{\frac{3 \theta+1}{2}}$. This completes the proof.

\section{Stability}

We consider a solitary wave solution of (1.6) of the form $u(x, y, t)=\varphi_{c}(x-c t, y)$, vanishing at infinity, where $c>0$ is the wave velocity; then $\varphi_{c}$ satisfies

$$
-c \partial_{x}^{2} \varphi_{c}+(c-\varepsilon) \varphi_{c}+\beta \partial_{y}^{2} \varphi_{c}-F\left(\varphi_{c}\right)=0 .
$$

Using some Pohozaev-type identities, one can easily show that there is no solitary wave solution of (1.6) if $c<\varepsilon$ or $\beta>0$.

Existence of solutions for (3.1) is well-known. It follows, for example, from the results of Berestycki and Lions [4]. We are interested in the cylindrically symmetric positive solutions of (3.1). We recall the results in the following theorem.

THEOREM 3.1. Suppose that $f$ satisfies the assumptions stated at the beginning of Section 2. If $c>0$ and $\beta<0$, then equation (3.1) possesses a cylindrically symmetric positive solution $\varphi_{c} \in H^{1}\left(\mathbb{R}^{2}\right)$, which is called a ground state of (3.1). Moreover, $\varphi_{c} \in H^{\infty}\left(\mathbb{R}^{2}\right), \partial_{r} \varphi_{c}(r)<0$, for all $r \neq 0$ where $r=|(\sqrt{c} x, \sqrt{-\beta} y)|$, and there is a $\sigma>0$ such that for all $\alpha \in \mathbb{N}^{2}$ with $|\alpha| \leq 2,\left|\partial^{\alpha} \varphi_{c}(x, y)\right| \leq C_{\alpha} \mathrm{e}^{-\sigma r}$.

For the sake of simplicity, throughout this section we assume that $\beta=-1$. of

We note that the solution $\varphi_{c}$ of (3.1), given by Theorem 3.1, is the critical point

$$
S_{c}\left(\varphi_{c}\right)=E\left(\varphi_{c}\right)+c Q\left(\varphi_{c}\right)
$$

Now, we define the linearized operator $L_{c}$ around $\varphi_{c}$ by

$$
L_{c}=S_{c}^{\prime \prime}=E^{\prime \prime}+c Q^{\prime \prime}=-c \partial_{x}^{2}-\partial_{y}^{2}+c-\varepsilon-f^{\prime}\left(\varphi_{c}\right) .
$$

It is clear that $L_{c}$ is self-adjoint from $H^{1}\left(\mathbb{R}^{2}\right)$ into $H^{-1}\left(\mathbb{R}^{2}\right)$ and that $\mathcal{L}^{-1 / 2} L_{c} \mathcal{L}^{-1 / 2}$ is self-adjoint on $L^{2}\left(\mathbb{R}^{2}\right)$. One can easily see that the solution $\varphi_{c}$ satisfies

$$
L_{c} \partial_{x} \varphi_{c}=0 \quad \text { and } \quad L_{c} \partial_{y} \varphi_{c}=0 .
$$

We continue by stating our hypotheses on $\varphi_{c}$ and $L_{c}$ (see lemmas 3.2 and 3.6 below), which are as follows. 
Hypothesis 3.1. The ground state solution of (3.1) given by Theorem 3.1 is unique and the curve $c \mapsto \varphi_{c}$ defined for $c>\varepsilon$ is $C^{1}$ with values in $H^{2}\left(\mathbb{R}^{2}\right)$. Moreover there are positive constants $C$ and $\varrho$ such that

$$
\left|\frac{\mathrm{d} \varphi_{c}}{\mathrm{~d} c}(x, y)\right| \leq C \mathrm{e}^{-\varrho|(x, y)|},
$$

for all $(x, y) \in \mathbb{R}^{2}$ and $c>\varepsilon$.

Hypothesis 3.2. The operator $L_{c}$, for $c>\varepsilon$, has a unique simple negative eigenvalue $\lambda_{c}$ with a corresponding cylindrically symmetric positive eigenfunction $\chi_{c}$ such that for all $(x, y) \in \mathbb{R}^{2}$,

$$
\left|\chi_{c}(x, y)\right| \leq C_{c} \mathrm{e}^{-\rho|(x, y)|},
$$

for some positive constants $C_{c}$ and $\rho$. In addition, the mapping $c \mapsto \chi_{c}$ is continuous with values in $H^{2}\left(\mathbb{R}^{2}\right)$. Furthermore the essential spectrum of $L_{c}$ is positive and bounded away from zero and the null space of $L_{c}$ is spanned by $\partial_{x} \varphi_{c}$ and $\partial_{y} \varphi_{c}$.

The following lemma considers the power-law nonlinearity $f(s)=s^{p+1} /(p+1)$.

Lemma 3.2. Let $p \geq 1$ be an integer and $f(u)=u^{p+1} /(p+1)$. Then hypotheses 3.1 and 3.2 are satisfied.

REMARK 3.3. It is noteworthy that if $\psi$ satisfies

$$
-\Delta \psi+\psi-\frac{\psi^{p+1}}{p+1}=0
$$

and the operator $L_{1}=-\Delta+1-\psi^{p}$ satisfies Hypothesis 3.2, i.e. $L_{1}$ has a unique simple negative eigenvalue $\lambda$ with a corresponding radially symmetric positive eigenfunction $\chi_{1}$, then the operator $L_{c}=-c \partial_{x}^{2}-\partial_{y}^{2}+c-\varepsilon-\varphi_{c}^{p}$ satisfies Hypothesis 3.2. More precisely, the unique simple negative eigenvalue of $L_{c}$ is a multiple of $\lambda$ and the cylindrically symmetric positive eigenfunction of $L_{c}$ is a dilation of $\chi_{1}$.

\section{Proof of Lemma 3.2.}

Proof. The regularity of $\varphi_{c}$ with respect to $c$ follows from Theorem 3.1 (see (3.9)). Uniqueness of the ground state was proved in [24]. We show that $\partial_{x} \varphi_{c}$ and $\partial_{y} \varphi_{c}$ are the only zero modes of $L_{c}$. By Remark 3.3 , it is enough to prove it for the operator

$$
L_{1}=-\Delta+1-\psi^{p} .
$$

We can use the same argument as in $[24,35]$ and see that any zero mode of $L_{1}$ can be decomposed into a series of radial functions multiplied by spherical harmonics. Since each one of $\partial_{x} \varphi_{c}$ and $\partial_{y} \varphi_{c}$ corresponds to spherical harmonics of degree 1, it follows that the only other possible zero modes correspond to spherical harmonics of degree zero. Thus, it is sufficient to show that $L_{1}$ has no radial zero mode, or more precisely the solution of

$$
u_{r r}+\frac{1}{r} u_{r}-u+u^{p}=0,
$$

where $r=|(x, y)|$, with $u(0)=1$ and $u^{\prime}(0)=0$, does not vanish at infinity. But Kwong in [24] showed that if $u(r, \alpha)$ is the solution of (3.4) with $u_{r}(0, \alpha)=0$ and $u(0, \alpha)=\alpha$, 
and if $\alpha_{0}$ is the initial value generating the ground state, then $u_{\alpha}\left(r, \alpha_{0}\right) \rightarrow+\infty$ as $r \rightarrow+\infty$. On the other hand, $u_{\alpha}\left(r, \alpha_{0}\right)$ satisfies $u(0)=1$ and $u^{\prime}(0)=0$; hence this solution does not vanish at infinity.

The properties of Hypothesis 3.2 are proved in [36, Proposition 4.2].

Now we examine the relation between the convexity properties of the function $d(c)$ and the properties of the functional $E$ near the critical point $\varphi_{c}$ under suitable constraints. Indeed by defining $\psi_{\omega}$ in the form

$$
\psi_{\omega}=\varphi_{c}+s(\omega) \chi_{c},
$$

where $s(c)$ is an appropriate function, we can prove following theorem exactly as $[8$, Theorem 3.1] or [32, Theorem 2.3].

TheOREM 3.3. If $d^{\prime \prime}(c)<0$, then there exists a curve $\omega \mapsto \psi_{\omega}$ passing through $\varphi_{c}$, lying on the surface $Q(u)=Q\left(\varphi_{c}\right)$, and on which $E(u)$ has a strict local maximum at $u=\varphi_{c}$.

We also denote the tubular neighborhoods of the orbit generated by a solitary wave by

$$
U_{\epsilon}=\left\{u \in H^{1}\left(\mathbb{R}^{2}\right) ; \inf _{z \in \mathbb{R}^{2}}\left\|u-\varphi_{c}(\cdot-z)\right\|_{1}<\epsilon\right\}
$$

and

$$
U_{\epsilon}^{s}=\left\{u \in U_{\epsilon} ; u \text { is } y \text {-cylindrically symmetric }\right\}
$$

where $\epsilon>0$.

Lemma 3.4. Fix $c>\varepsilon$ and let $\varphi_{c}$ be a solitary wave given in Theorem 3.1. Then, there exist an $\epsilon>0$ and a $C^{1}-$ map $\zeta: U_{\epsilon} \rightarrow \mathbb{R}^{2}$ such that for all $u \in U_{\epsilon}$ and $z \in \mathbb{R}^{2}$,

(i) $\left\langle u(\cdot+\zeta(u)), \partial_{x} \varphi_{c}\right\rangle=\left\langle u(\cdot+\zeta(u)), \partial_{y} \varphi_{c}\right\rangle=0$,

(ii) $\zeta(u(\cdot+z))=\zeta(u)-z$.

(iii) Moreover, if $u \in U_{\epsilon}^{s}$ then $\zeta(u)=\left(\zeta_{0}(u), 0\right)$, where

$$
\zeta_{0}^{\prime}(u)=\frac{\partial_{x} \varphi_{c}(\cdot-\zeta(u))}{\left\langle u, \partial_{x}^{2} \varphi_{c}(\cdot-\zeta(u))\right\rangle} .
$$

Proof. The proof of this lemma is well-known. In fact, one defines $F: H^{1}\left(\mathbb{R}^{2}\right) \times$ $\mathbb{R}^{2} \rightarrow \mathbb{R}^{2}$ by

$$
F(u, \zeta)=\int_{\mathbb{R}^{2}} u((x, y)+\zeta) \nabla \varphi_{c}(x, y) \mathrm{d} x \mathrm{~d} y,
$$

where $\zeta=\left(\zeta_{1}, \zeta_{2}\right)$. Then, taking into account that $\varphi_{c}$ is cylindrically symmetric (see Theorem 3.1), one can apply the Implicit Function Theorem to conclude the statements. For details we refer the reader to [8, Lemma 4.1].

We continue by defining for $u \in U_{\epsilon}^{s}$ the mapping

$$
\begin{aligned}
B(u) & =\vartheta(\cdot-\gamma(t), \cdot)-\langle\vartheta(\cdot-\gamma(t), \cdot), \mathcal{L} u\rangle \partial_{x} \mathcal{L}^{-1} \zeta_{0}^{\prime}(u) \\
& =\vartheta(\cdot-\gamma(t), \cdot)-\frac{\langle\vartheta(\cdot-\gamma(t), \cdot), \mathcal{L} u\rangle}{\left\langle u, \partial_{x}^{2} \varphi_{c}(\cdot-\gamma(t), \cdot)\right\rangle} \partial_{x}^{2} \partial_{x} \mathcal{L}^{-1} \varphi_{c}(\cdot-\gamma(t), \cdot),
\end{aligned}
$$


where $\gamma(t)=\zeta_{0}(u(t))$ and $\vartheta$ is defined by

$$
\vartheta=\left.\frac{\mathrm{d} \psi_{\omega}}{\mathrm{d} \omega}\right|_{\omega=c} .
$$

Now we can prove the following properties of $B$.

Lemma 3.5. For any small $\epsilon>0$, the mapping $B$ is a $C^{1}$-function from $U_{\epsilon}^{s}$ into $H^{1}\left(\mathbb{R}^{2}\right)$. In addition, the following statements hold:

(i) $B\left(\varphi_{c}\right)=\vartheta$;

(ii) $\langle B(u), \mathcal{L} u\rangle=0$, for all $u \in U_{\epsilon}^{s}$;

(iii) $B$ commutes with translations in x-variable.

Proof. The proof of this lemma is standard by using Lemma 3.4. For details we refer the reader to [8, Proposition 4.1].

Hypothesis 3.4. We assume that $\mathcal{L}\left(\frac{\mathrm{d} \varphi_{c}}{\mathrm{~d} c}\right) \in L^{1}\left(\mathbb{R}^{2}\right)$.

Lemma 3.6. If $f(u)=u^{p+1} /(p+1)$ with $p \geq 1$, then Hypothesis 3.4 is satisfied.

Proof. First we note from Theorem 3.1 that $\partial^{\alpha} \varphi_{c} \in L^{1}\left(\mathbb{R}^{2}\right)$, for all $\alpha \in \mathbb{N}^{2}$ with $|\alpha| \leq 2$. An argument similar to [7] and [14] shows from

$$
\varphi_{c}=K_{c} * f\left(\varphi_{c}\right), \quad \widehat{K}_{c}(\xi, \eta)=\frac{1}{c \xi^{2}+\eta^{2}+c-\varepsilon},
$$

that $\partial^{\alpha} \varphi_{c} \in L^{1}\left(\mathbb{R}^{2}\right)$ for all $\alpha \in \mathbb{N}_{0}^{3}$ with $|\alpha| \leq 3$. Hence the proof follows from the fact that if $\varphi_{c}$ is a ground state of $(3.1)$, then $\varphi_{c} \in H^{\infty}\left(\mathbb{R}^{2}\right)$ and

$$
\psi(x, y)=(c-\varepsilon)^{-1 / p} \varphi_{c}\left(\sqrt{\frac{c}{c-\varepsilon}} x, \frac{1}{\sqrt{c-\varepsilon}} y\right)
$$

satisfies

$$
-\Delta \psi+\psi-f(\psi)=0
$$

Lemma 3.7. Let $f \in C^{2}(\mathbb{R})$ satisfy the assumptions stated at the beginning of Section 2. Then $\mathcal{L} \vartheta \in L^{1}\left(\mathbb{R}^{2}\right)$.

Proof. We recall that $\vartheta$ is defined by

$$
\vartheta=\frac{\mathrm{d} \varphi_{c}}{\mathrm{~d} c}+s^{\prime}(c) \chi_{c}
$$

for some real-valued function $s$. We have from Hypothesis 3.2 and Lemma 3.6 that $\mathcal{L}\left(\mathrm{d} \varphi_{c} / \mathrm{d} c\right), \chi_{c} \in L^{1}\left(\mathbb{R}^{2}\right)$. On the other hand, since $\chi_{c}$ satisfies $\mathcal{L} \chi_{c}=\lambda \chi_{c}$, for some $\lambda<0$, then $\chi_{c}$ satisfies

$$
\chi_{c}=\widetilde{K}_{c} *\left(f^{\prime}\left(\varphi_{c}\right) \chi_{c}\right), \quad \widehat{\widetilde{K}}_{c}(\xi, \eta)=1 /\left(c \xi^{2}+\eta^{2}+c-\varepsilon-\lambda\right) .
$$

Hence by the aforementioned argument in the proof of Lemma 3.6, we obtain that $\partial_{x}^{2} \chi_{c} \in L^{1}\left(\mathbb{R}^{2}\right)$. This completes the proof.

We define the stability of solitary wave in the usual way. 
DeFINITION 3.8. Let $\varphi_{c}$ be a solitary wave solution of (1.6). We say that $\varphi_{c}$ is orbitally (or nonlinearly) stable if for all $\epsilon>0$, there is a $\delta>0$ such that for any $u_{0} \in H^{s}\left(\mathbb{R}^{2}\right), s>1$, with $\left\|u_{0}-\varphi_{c}\right\|_{1} \leq \delta$, the corresponding solution $u(t)$ of (1.6) with $u(0)=u_{0}$ satisfies

$$
\sup _{t \geq 0} \inf _{z \in \mathbb{R}^{2}}\left\|u(t)-\varphi_{c}(\cdot-z)\right\|_{1} \leq \epsilon
$$

Otherwise, we say that $\varphi_{c}$ is nonlinearly unstable.

THEOREM 3.9. Suppose that $f$ satisfies the assumptions stated at the beginning of Section 2. Let $c>\varepsilon$ and $\varphi_{c}$ be a solution of (3.1) given by Theorem 3.1, and also assume that hypotheses 3.1, 3.2, and 3.4 hold. Then $\varphi_{c}$ is stable if and only if $d^{\prime \prime}(c)>$ 0 , where

$$
d(c)=E\left(\varphi_{c}\right)+c Q\left(\varphi_{c}\right)
$$

Proof of stability is exactly the same as in $[8,20,32]$, so we omit it and we only prove instability.

Lemma 3.10. Suppose that $c>\varepsilon$ and $d^{\prime \prime}(c)<0$. Let $\varphi_{c}$ be a solitary wave given in Theorem 3.1. Then there is a small enough $\epsilon>0$ such that for any $u \in U_{\epsilon}^{s}$ which is not a translation of $\varphi_{c}$ and satisfies $Q(u)=Q\left(\varphi_{c}\right)$, there is a $\lambda=\lambda(u) \in(-\epsilon, \epsilon)$ such that

$$
E\left(\varphi_{c}\right)<E(u)+\lambda\left\langle E^{\prime}(u), B(u)\right\rangle .
$$

Proof. The proof follows from Taylor's theorem. For details see [15, Lemma 3.11] or [32, Lemma 4.3].

The following lemma has a key role in the proof of instability. The proof is similar to Lemma 4.4 in [32].

Lemma 3.11. Let $c>\varepsilon$ and $\varphi_{c}$ be a corresponding solitary wave given in Theorem (3.1). Assume $d^{\prime \prime}(c)<0$. Then, the curve $c \mapsto \psi_{c}$ defined in Theorem 3.3 satisfies $E\left(\psi_{\omega}\right)<E\left(\varphi_{c}\right)$ for $\omega \neq c, Q\left(\psi_{\omega}\right)=Q\left(\varphi_{c}\right)$, and $\left\langle E^{\prime}\left(\psi_{\omega}\right), B\left(\psi_{\omega}\right)\right\rangle$ changes sign as $\omega$ passes through $c$.

Now we turn our attention to complete the proof of instability of Theorem 3.9.

Let $\epsilon>0$ be sufficiently small as in Lemma $3.4, U_{\epsilon}^{s}$ be the corresponding tubular neighborhood and a cylindrically symmetric data $u_{0}=\psi_{\omega}$ with $\omega$ close to $c$ (in $H^{1}\left(\mathbb{R}^{2}\right)$ norm), so that $u_{0} \in U_{\epsilon}^{s} \cap H^{s}\left(\mathbb{R}^{2}\right)$ with $s>1, E\left(u_{0}\right)<E\left(\varphi_{c}\right)$, and $\left\langle E^{\prime}\left(u_{0}\right), B\left(u_{0}\right)\right\rangle>0$. By Theorem 2.1, there exists a $T^{*}>0$ and a solution $u(t) \in C\left(\left[0, T^{*}\right) ; H^{s}\left(\mathbb{R}^{2}\right)\right)$ of $(1.6)$ with $u(0)=u_{0}$. Let $T^{*}$ be the maximal time for which $u(t) \in C\left(\left[0, T^{*}\right) ; H^{s}\left(\mathbb{R}^{2}\right)\right)$. Note that since $u_{0}$ belongs to $U_{\epsilon}^{s}$ so belongs $u(t)$, for $t \in[0, T]$ with $T \leq T^{*}$ (since the gBBMZK equation is invariant from $y$ to $-y$ ). We want to show that $T<+\infty$, which means that $u(t)$ eventually leaves $U_{\epsilon}^{s}$.

Define the Lyapunov function

$$
A(t)=\int_{\mathbb{R}^{2}} \partial_{x}^{-1} u(x, y, t) \mathcal{L} \vartheta(x-\gamma(t), y) \mathrm{d} x \mathrm{~d} y .
$$


From the Hölder inequality, Lemma 3.7, and Theorem 2.10, we have

$$
|A(t)| \leq\left|\partial_{x}^{-1} u(t)\right|_{\infty}|\mathcal{L} \vartheta|_{1} \lesssim(1+t)^{\frac{3 \theta+1}{2}} .
$$

On the other hand, we see from $u_{t}-\mathcal{L}^{-1} \partial_{x} E^{\prime}(u)=0$ and the definition of $B$ that

$$
\begin{aligned}
\frac{\mathrm{d}}{\mathrm{d} t} A(t) & =\left\langle\partial_{x}^{-1} \frac{\mathrm{d} u}{\mathrm{~d} t}, \mathcal{L} \vartheta(\cdot-\gamma(t), \cdot)\right\rangle-\left\langle\partial_{x}^{-1} u, \partial_{x}(\mathcal{L} \vartheta(\cdot-\gamma(t), \cdot))\right\rangle\left\langle\zeta_{0}^{\prime}(u(t)), \frac{\mathrm{d} u}{\mathrm{~d} t}\right\rangle \\
& =\left\langle E^{\prime}(u(t)), \vartheta(\cdot-\gamma(t), \cdot)\right\rangle-\langle u, \mathcal{L} \vartheta(\cdot-\gamma(t), \cdot)\rangle\left\langle\partial_{x} \mathcal{L}^{-1} \zeta_{0}^{\prime}(u(t)), E^{\prime}(u(t))\right\rangle \\
& =\left\langle E^{\prime}(u(t)), B(u(t))\right\rangle .
\end{aligned}
$$

Since $E$ is conserved, we obtain from Lemma 3.10 that

$$
\lambda(u(t))\left\langle E^{\prime}(u(t)), B(u(t))\right\rangle>E\left(\varphi_{c}\right)-E\left(u_{0}\right)=: C_{0}>0 .
$$

So, since $\left\langle E^{\prime}\left(u_{0}\right), B\left(u_{0}\right)\right\rangle>0$, we deduce that $\lambda(u(t))>0$ and we may choose (if necessary) $\epsilon>0$ small enough such that $|\lambda(u(t))|<1$ as long as $u(t) \in U_{\epsilon}^{s}$. Therefore, from (3.14), we have for $0 \leq t \leq T$,

$$
0<C_{0}<\left|\left\langle E^{\prime}(u(t)), B(u(t))\right\rangle\right|=\left|\frac{\mathrm{d}}{\mathrm{d} t} A(t)\right| .
$$

As a consequence of (3.13) and (3.15) we deduce that $T$ is necessarily finite, i.e., $u(t)$ must exits $U_{\epsilon}^{s}$ in a finite time. This proves the theorem.

3.1. Application to power-law nonlinearity. Now we apply our stability results for the case $f(u)=u^{p+1} /(p+1)$. It is already seen in (3.9) that if $\varphi_{c}$, with $c>\varepsilon$, is the positive cylindrical solution of (3.1), then $\psi$ defined in (3.9) satisfies (3.10). lemmas 3.2 and 3.6 show that hypotheses 3.1, 3.2, and 3.4 hold in this case.

A straightforward calculation reveals from the Pohozaev-type identities that

$$
d(c)=c \int_{\mathbb{R}^{2}}\left(\partial_{x} \varphi_{c}\right)^{2} \mathrm{~d} x \mathrm{~d} y
$$

Hence

$$
d(c)=\sqrt{c}(c-\varepsilon)^{2 / p} \int_{\mathbb{R}^{2}} \psi_{x}^{2} \mathrm{~d} x \mathrm{~d} y
$$

so that

$$
d^{\prime \prime}(c)=\frac{c^{3 / 2}(c-\varepsilon)^{2 / p-2}}{4 p^{2}} \mathscr{N}(\varepsilon, c, p)
$$

where

$$
\mathscr{N}(\varepsilon, c, p)=c^{2}\left(16-p^{2}\right)+2 c p(p \varepsilon-4)-p^{2} \varepsilon .
$$

Therefore we get the fact that $\varphi_{c}$ is stable if one of the following cases occurs, and is unstable otherwise:

(i) $p=4, \varepsilon>1$, and $c>\max \left\{\varepsilon, \frac{\varepsilon}{2(\varepsilon-1)}\right\}$,

(ii) $p<4, p \varepsilon=4$, and $c>\max \left\{\varepsilon, \sqrt{\frac{\varepsilon}{\varepsilon^{2}-1}}\right\}$, 
(iii) $p<4, p \varepsilon \neq 4$, and $c>\max \left\{\varepsilon, \frac{4-p \varepsilon+\sqrt{(p \varepsilon-4)^{2}+\varepsilon\left(16-p^{2}\right)}}{16-p^{2}} p\right\}$,

(iv) $p>4, p \varepsilon<4$, and $\{c>\varepsilon\} \cap\left\{c<\frac{p \varepsilon-4+\sqrt{p^{2} \varepsilon(\varepsilon-1)-8 p \varepsilon+16(\varepsilon+1)}}{p^{2}-16} p\right\}$,

(v) $p>4, p \varepsilon \neq 4$, and $\{c>\varepsilon\} \cap\left\{a_{1}(p)<c<a_{2}(p)\right\}$, where

$$
a_{1}(p)=\frac{p \varepsilon-4-\sqrt{p^{2} \varepsilon(\varepsilon-1)-8 p \varepsilon+16(\varepsilon+1)}}{p^{2}-16} p
$$

and

$$
a_{2}(p)=\frac{p \varepsilon-4+\sqrt{p^{2} \varepsilon(\varepsilon-1)-8 p \varepsilon+16(\varepsilon+1)}}{p^{2}-16} p .
$$

In particular, when $\varepsilon=1$, we observe that $\varphi_{c}$, with $c>1$, is stable for $p \leq 2$ or $p \in[3,4)$ and

$$
c>\left(\frac{4-p+\sqrt{8-2 p}}{16-p^{2}}\right) p,
$$

and unstable otherwise. Figure 3.1 illustrates the regions of instability of solitary waves of (1.6) for $\varepsilon=0.2$ and $p=1, \cdots, 5$.

3.2. Stability of solitary waves of the gZK, gBBM-ZK, and 2D-gBBM equations. We are going to compare the stability of solitary waves of (1.6) to (1.5) and (1.7). We note that if $\varphi_{c}(x-c t, y)$ is a ground state of (1.7), then $\varphi_{c}$ satisfies

$$
(c-\varepsilon) \varphi_{c}-c \Delta \varphi_{c}-F\left(\varphi_{c}\right)=0 .
$$

As we mentioned before, when $\varepsilon=1$, the stability and the instability of solitary wave solutions of (1.7) have been investigated by de Bouard [12]. We consider the homogeneous nonlinearity $f(u)=u^{p+1} /(p+1)$. In [12], it was proved that the solitary waves of (1.7) is stable if $d_{2 D-B B M}^{\prime \prime}(c)>0$, and is unstable if $d_{2 D-B B M}^{\prime \prime}(c)<0$, where

$$
d_{2 D-B B M}(c)=\frac{c}{2}(c-\varepsilon)^{2 / p}|\nabla \psi|_{2}^{2},
$$

and $\psi$ is the radial positive solution of (3.10).

It was also proved in [12] that the solitary wave $\varphi_{c}$, with $c>0$, of (1.5) is stable if $d_{Z K}^{\prime \prime}(c)>0$, and is unstable if $d_{Z K}^{\prime \prime}(c)<0$, where

$$
d_{Z K}(c)=\frac{1}{2} c^{2 / p}|\nabla \psi|_{2}^{2},
$$

and $\psi$ is the radial positive solution of (3.10). More precisely, the solitary wave $\varphi_{c}$ is stable if $p<2$, while it is unstable if $p>2$. When $p=2$, the stability/instability of $\varphi_{c}$ is still unknown (see figure 3.2).

For the 2D-gBBM equation, one can observe for fixed $\varepsilon>0$ that there exists $p_{1}^{*}=p_{1}^{*}(\varepsilon) \in(2,3]$ such that the solitary wave $\varphi_{c}$ is stable if $c>\varepsilon$ and $p \leq p_{1}^{*}$. For $p \geq p_{1}^{*}$, a straightforward calculation reveals that there exists $c^{*}=c_{p, \varepsilon}^{*}>0$ such that the solitary wave $\varphi_{c}$ is stable if and only if $c>c^{*}$. Figure 3.1 illustrates the region of stability of $\varphi_{c}$ for $\varepsilon=0.2$.

Our results above show that for the fixed $\varepsilon>0$, there exists $p_{2}^{*}=p_{1}^{*}(\varepsilon) \in(2,3] \mathrm{such}$ that the solitary wave $\varphi_{c}$ is stable if $c>\varepsilon$ and $p \leq p_{2}^{*}$. On the other hand, for $p \geq p_{2}^{*}$, a straightforward calculation reveals that there exists $c^{*}=c_{p, \varepsilon}^{*}>0$ such that the solitary 

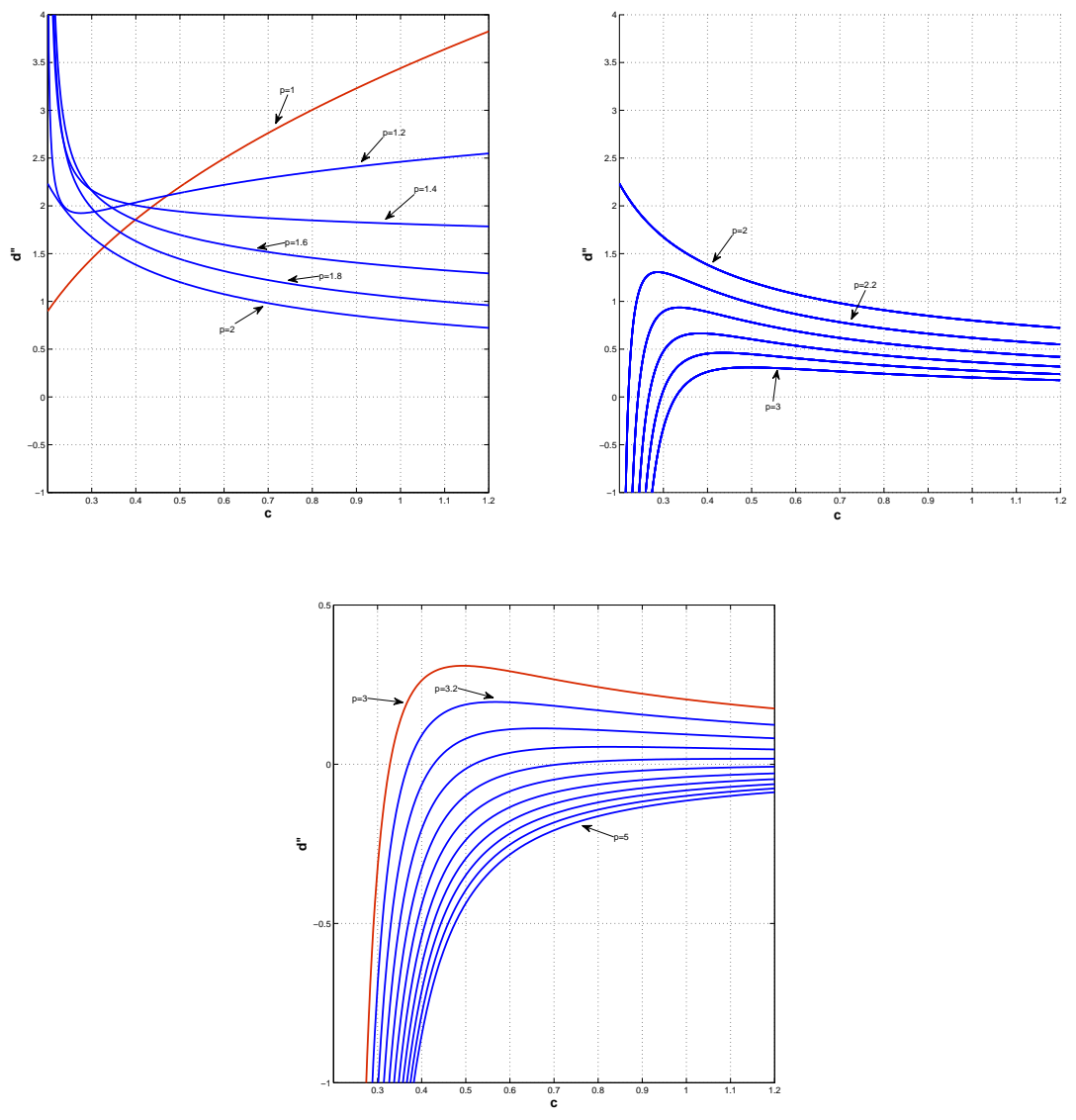

FiG. 3.1. The region of stability of the ground states of the $g B B M-Z K$ equation, $p=1-2,2-$ $3,3-5$, and $\varepsilon=0.2$.
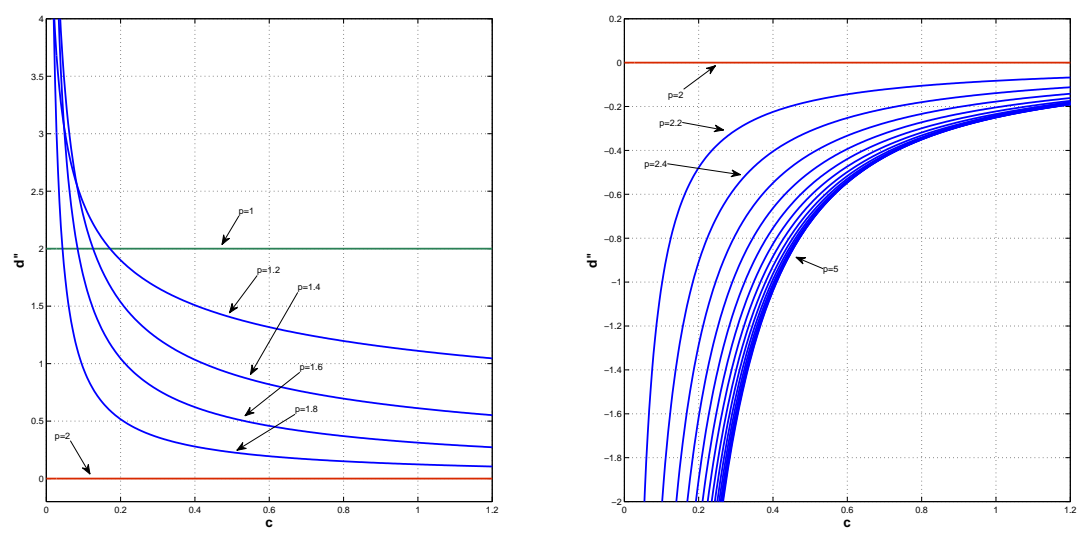

FIG. 3.2. The region of stability of solitary waves of the $g Z K$ equation for $p=1-2,2-5$. 

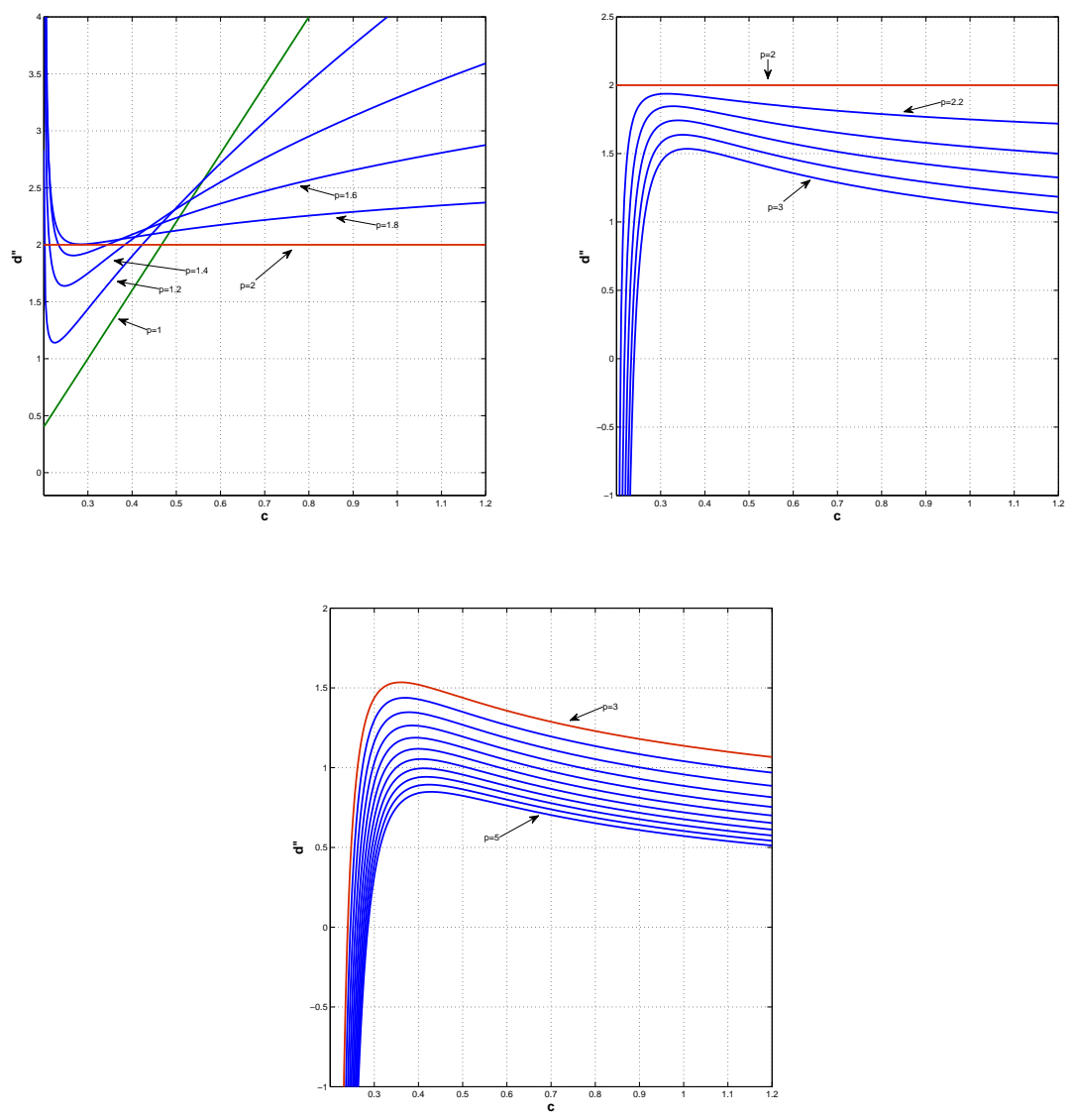

FIG. 3.3. The region of stability of solitary waves of the $2 D-g B B M$ equation for $p=1-2,2-$ $3,3-5$, and $\varepsilon=0.2$.

wave $\varphi_{c}$ is stable if and only if $c>c^{*}$. Figure 3.3 illustrates the region of stability of $\varphi_{c}$ for $\varepsilon=0.2$.

These results show that $p_{1}^{*}>p_{2}^{*}$, so that the region of stability of solitary waves of the 2D-gBBM equation is bigger than the stability regions of solitary waves for the gBBM-ZK equation. Also the region of stability of solitary waves of the gBBM-ZK equation is bigger than the stability regions of solitary waves for the gZK equation. Therefore, the 2D-gBBM equation has better behavior in stability of solitary waves than the gZK and the gBBM-ZK equations.

3.3. Effects of transport equation. Since equations (1.7) and (1.6) are obtained by combining the transport equation and the gZK equation, we investigate here the effects of the transport equation on the stability of solitary waves of equations (1.7) and (1.6). Actually, we want to see the effects of perturbations of the parameter $\varepsilon$ on the region of stability of solitary waves of the gBBM-ZK and the 2D-gBBM equations.

One can observe from the results above and figures 3.5 and 3.4 that for a fixed $p>2$, the region of stability of solitary waves, for both equations (1.6) and (1.7), 

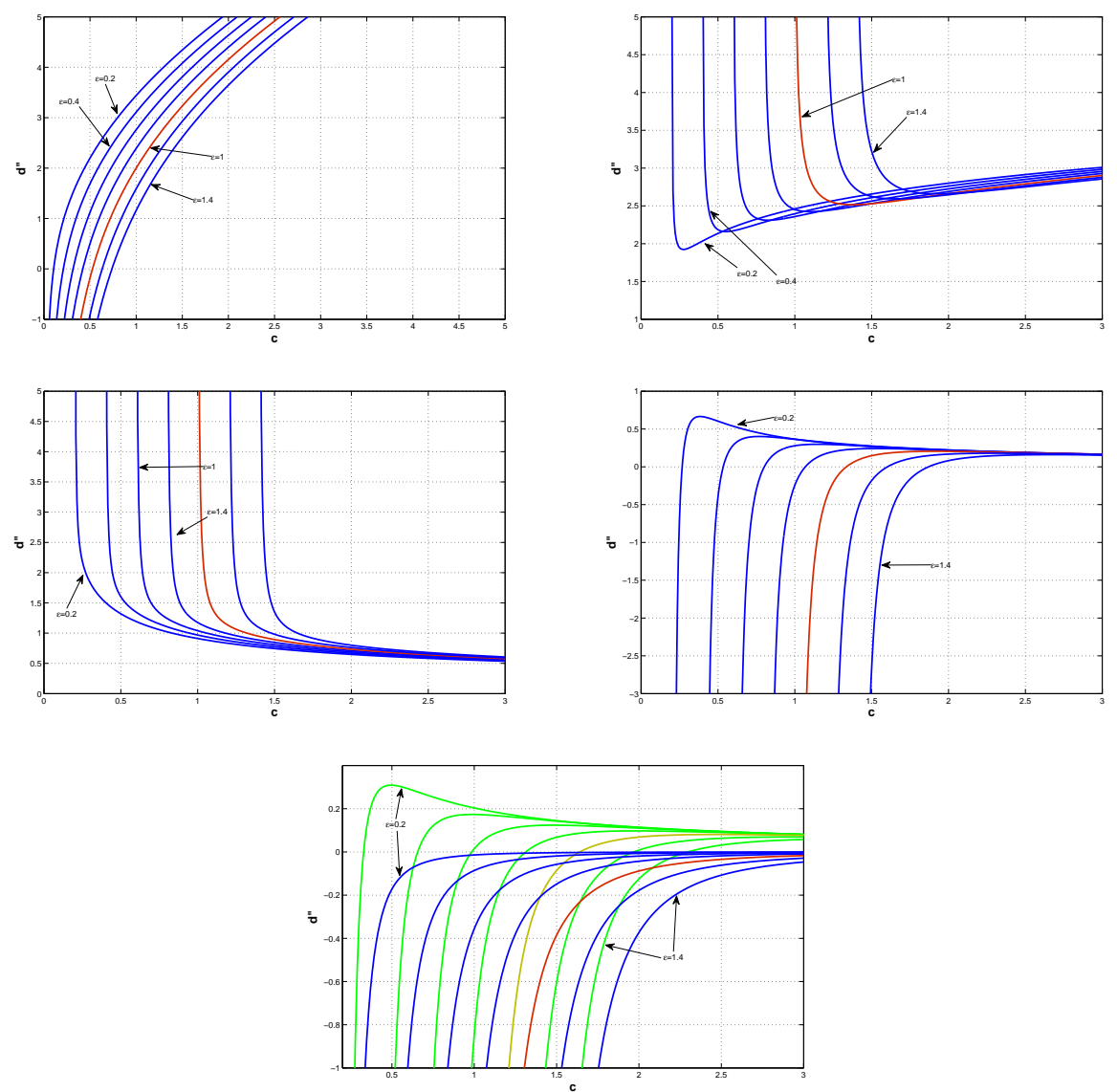

FIG. 3.4. The region of stability of solitary waves of the $g B B M-Z K$ equation, from left to right and then from top to bottom, for $p=1,1.2,1.9,2.6$. Green and blue correspond respectively to $p=3$ and $p=4$ in the last figure.

becomes bigger when $\varepsilon>0$ tends to zero.

REMARK 3.5. One can easily observe that the transport equation with the parameter $\varepsilon>0$ does not affect the region of stability of the gZK equation. Indeed, in this case the solitary wave $\varphi_{c}$, with $c>\varepsilon$, is stable if $p<2$ and is unstable if $p>2$.

Acknowledgment. The author would like to thank Luc Molinet, Jerry L. Bona, and Felipe Linares for their suggestions and comments. The author also wishes to thank the unknown referees for their careful reading and suggestions which helped to improve the paper. 

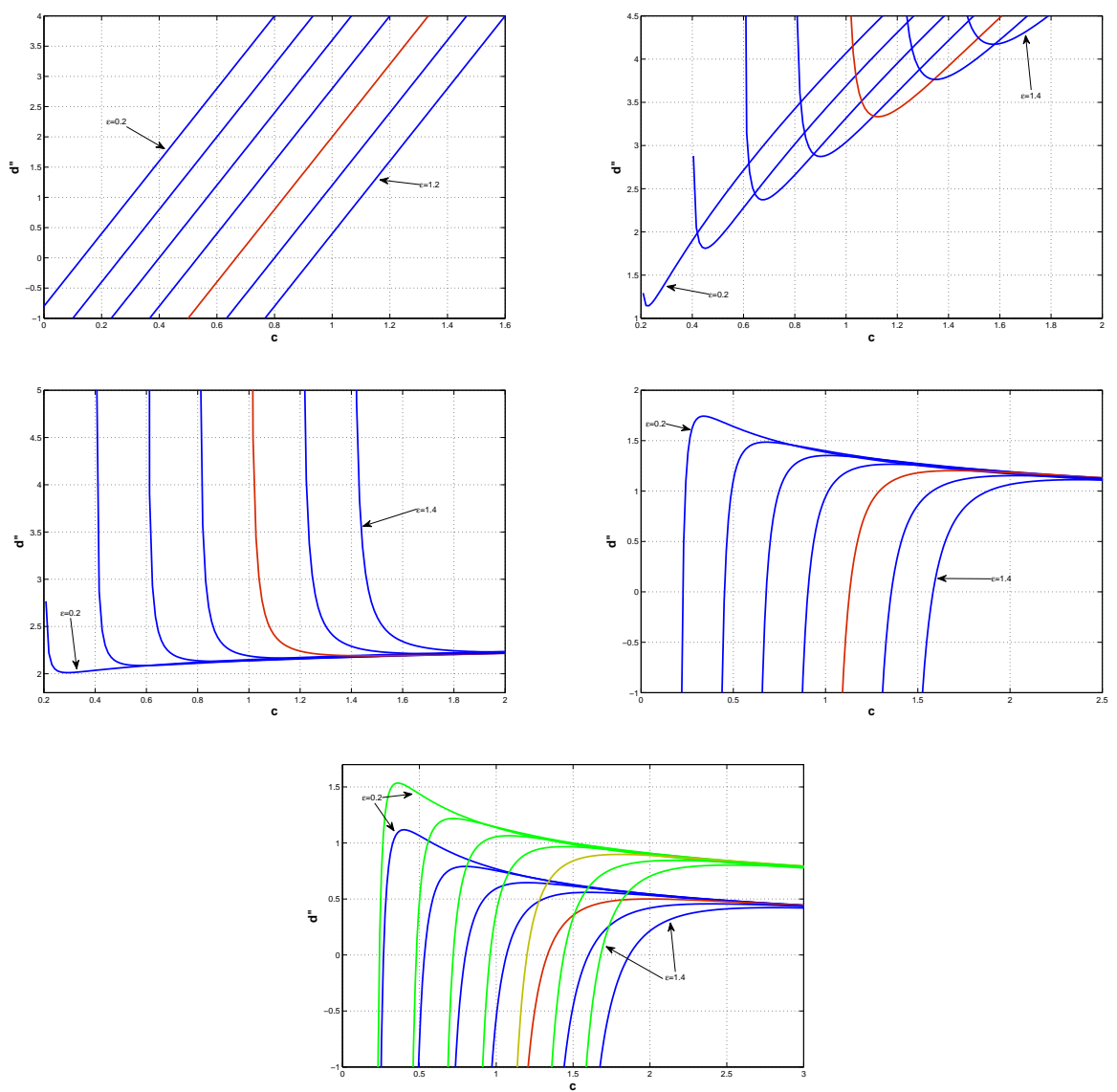

FIG. 3.5. The region of stability of solitary waves of the 2D-gBBM equation, from left to right and then from top to bottom, for $p=1,1.2,1.9,2.6$. Green and blue correspond respectively to $p=3$ and $p=4$ in the last figure.

\section{REFERENCES}

[1] M.A. Abdou, The extended F-expansion method and its application for a class of nonlinear evolution equations, Chaos, Solitons and Fractals, 31, 95-104, 2007.

[2] T.B. Benjamin, J.L. Bona, and J.J. Mahony, Model equations for long waves in nonlinear dispersive systems, Philos. Trans. Roy. Soc. London Ser. A, 272, 47-78, 1972.

[3] H. Berestycki, T. Gallouët, and O. Kavian, Équations de champs scalaires euclidiens non linéaires dans le plan, C.R. Acad. Sci. Paris Sér. I Math., 297, 307-310, 1983.

[4] H. Berestycki and P.L. Lions, Nonlinear scalar field equation, Arch. Ratio. Mech. Anal., 82, 313-376, 1983.

[5] H.A. Biagioni and F. Linares, Well-posedness results for the modified Zakharov-Kuznetsov equation, Nonlinear Equ. Meth. Model. Appl., Progr. Nonlinear Diff. Equ. Appl., Birkhuser, Basel, 54, 181-189, 2003.

[6] P. Biler, J. Dziubanski, and W. Hebisch, Scattering of small solutions to generalized BenjaminBona-Mahony equation in several space dimensions, Commun. Part. Diff. Equ., 17, 17371758, 1992.

[7] J.L. Bona and Y.A. Li, Decay and analyticity of solitary waves, J. Math. Pures Appl., 76, 377-430, 1997.

[8] J.L. Bona, P.E. Souganidis, and W.A. Strauss, Stability and instability of solitary waves of Korteweg-de Vries equation, Proc. Royal Soc. London Ser. A, 411, 395-412, 1987. 
[9] J. Bourgain, Fourier transform restriction phenomena for certain lattice subsets and applications to nonlinear evolution equations. I. Schrödinger equations, Geom. Funct. Anal., 3, 107-156, 1993.

[10] J. Bourgain, Fourier transform restriction phenomena for certain lattice subsets and applications to nonlinear evolution equations. II. The KdV-equation, Geom. Funct. Anal., 3, 209-262, 1993.

[11] J. Bourgain, On the Cauchy problem for the Kadomtsev-Petviashvili equation, Geom. Funct. Anal., 3, 315-341, 1993.

[12] A. de Bouard, Stability and instability of some nonlinear dispersive solitary waves in higher dimension, Proc. Roy. Soc. Edinburgh Sect. A, 126, 89-112, 1996.

[13] A. Esfahani, Remarks on a two dimensional BBM type equation, Commun. Pure Appl. Anal., 11, 1111-1127, 2012.

[14] A. Esfahani, Decay properties of the traveling waves of the rotation-generalized KadomtsevPetviashvili equation, J. Phys. A: Math. Theor., 43, 395201, 2010.

[15] A. Esfahani and A. Pastor, Instability of solitary wave solutions for the generalized BO-ZK equation, J. Diff. Equ., 247, 3181-3201, 2009.

[16] A.V. Faminskii, The Cauchy problem for the Zakharov-Kuznetsov equation, Diff. Equ., 31, 1002-1012, 1995.

[17] L.G. Farah, F. Linares, and A. Pastor, A note on the 2D generalized Zakharov-Kuznetsov equation: Local, global, and scattering results, J. Diff. Equ., 253, 2558-2571, 2012.

[18] A. Friedman, Partial Differential Equations, Holt, Rinehart and Winston, New York, 1969.

[19] J. Ginibre, Le problème de Cauchy pour des EDP semi-linéaires périodiques en variables d'espace (d'après Bourgain), Astérisque, 1996, Exp. 796(4), 163-187, Séminaire Bourbaki, $1994 / 95$.

[20] M. Grillakis, J. Shatah, and W. Strauss, Stability theory of solitary waves in the presence of symmetry. I, J. Funct. Anal., 74, 160-197, 1987.

[21] R.J. Iório, $K d V, B O$ and friends in weighted Sobolev spaces, in: Functional-Analytic Methods for Partial Differential Equations, Tokyo, 1989, in: Lecture Notes in Math., SpringerVerlag, Berlin, 1450, 104-121, 1990.

[22] M.A. Johnson, The transverse instability of periodic waves in Zakharov-Kuznetsov type equations, Stud. Appl. Math., 124, 323-345, 2010.

[23] T. Kato and G. Ponce, Commutator estimates and the Euler and Navier-Stokes equations, Commun. Pure Appl. Math., 41, 891-907, 1988.

[24] M.K. Kwong, Uniqueness of positive radial solutions of $\Delta u-u+u^{p}$ in $\mathbb{R}^{n}$, Arch. Ratio. Mech. Anal., 105, 243-266, 1989.

[25] F. Linares and A. Pastor, Well-posedness for the two-dimensional modified ZakharovKuznetsov equation, SIAM J. Math. Anal., 41, 1323-1339, 2009.

[26] F. Linares and A. Pastor, Local and global well-posedness for the $2 D$ generalized ZakharovKuznetsov equation, J. Funct. Anal., 260, 1060-1085, 2011.

[27] Y. Liu and M.M. Tom, Blow-up and instability of a regularized long-wave-KP equation, Diff. Integral Equ., 19, 1131-1152, 2003.

[28] A. Pazy, Semigroups of Linear Operators and Applications to Partial Differential Equations, Springer-Verlag, 1983.

[29] F. Ribaud and S. Vento, Well-posedness results for the three-dimensional Zakharov-Kuznetsov equation, SIAM J. Math. Anal., 44, 2289-2304, 2012.

[30] F. Ribaud and S. Vento, A note on the Cauchy problem for the $2 D$ generalized ZakharovKuznetsov equations, Comptes Rendus Math., 350, 499-503, 2012.

[31] I. Segal, Non-linear Semi-groups, Ann. of Math., 78, 339-364, 1963.

[32] P.E. Souganidis and W. Strauss, Instability of a class of dispersive solitary waves, Proc. Roy. Soc. Edinburgh A, 114, 195-212, 1990.

[33] E.M. Stein, Harmonic Analysis: Real-Variable Methods, Orthogonality, and Oscillatory Integrals, Princeton University Press, 1993.

[34] A.M. Wazwaz, Compact and noncompact physical structures for the ZK-BBM equation, Appl. Math. Comput., 169, 713-725, 2005.

[35] M.I. Weinstein, Modulational stability of ground states of nonlinear Schrödinger equations, SIAM J. Math. Anal., 16, 472-491, 1985.

[36] M.I. Weinstein, Lyapunov stability of ground states of nonlinear dispersive evolution equations, Commun. Pure Appl. Math., 39, 51-68, 1986. 